\title{
Research Article \\ Effects of Compound K-Distributed Sea Clutter on Angle Measurement of Wideband Monopulse Radar
}

\author{
Hong Zhu, Yujian Pan, Weiwei Wu, Ning Tai, and Naichang Yuan \\ College of Electronic Science and Engineering, National University of Defense Technology, Changsha 410073, China \\ Correspondence should be addressed to Hong Zhu; zhuhong13@nudt.edu.cn
}

Received 17 January 2017; Revised 8 April 2017; Accepted 20 April 2017; Published 24 May 2017

Academic Editor: Benjamin Ivorra

Copyright (c) 2017 Hong Zhu et al. This is an open access article distributed under the Creative Commons Attribution License, which permits unrestricted use, distribution, and reproduction in any medium, provided the original work is properly cited.

\begin{abstract}
The effects of compound $K$-distributed sea clutter on angle measurement of wideband monopulse radar are investigated in this paper. We apply the conditional probability density function (pdf) of monopulse ratio (MR) error to analyze these effects. Based on the angle measurement procedure of the wideband monopulse radar, this conditional pdf is first deduced in detail for the case of compound $K$-distributed sea clutter plus noise. Herein, the spatial correlation of the texture components for each channel clutter and the correlation of the texture components between the sum and difference channel clutters are considered, and two extreme situations for each of them are tackled. Referring to the measured sea clutter data, angle measurement performances in various $K$-distributed sea clutter plus noise circumstances are simulated, and the effects of compound $K$-distributed sea clutter on angle measurement are discussed.
\end{abstract}

\section{Introduction}

Monopulse, which uses the sum and difference beams to estimate the direction of arrival (DOA) of targets, is a matured technique of target angle measurement. Monopulse radars are widely used in target tracking area because of their superior angular accuracy and powerful antijamming performance $[1,2]$. However, when monopulse radar is used to track the targets on the sea, two problems occur. On the one hand, sea clutter lowers the signal-clutter-noise ratio (SCNR), which thereby decreases the radar detection probability and the angle measurement accuracy. On the other hand, multipath propagation makes the target and its image appear simultaneously in the main lobe of radar, which causes acute errors in the angle measurement of targets [2-4].

A complex target can be represented as a set of separate scattering centers. For wideband monopulse radar, since the range resolution is very high, these scattering centers can be effectively separated within different range resolution cells. Therefore, the wideband monopulse radar is used to restrain the angular glint effectively [5-7]. On the other hand, for the multipath propagation, the time delay of a target is smaller than its image. The wideband radar can use its advantage of high resolution to resolve the target and its image in the range profiles. Thus, the multipath interference is controlled significantly $[8,9]$. Due to all the advantages mentioned above, in recent years, the wideband monopulse radars are widely applied in various scenarios.

In the monopulse radar system, the angular information of a target is extracted from the monopulse ratio (MR), which is the ratio of the difference channel and the sum channel phasors. For the monopulse radar designers, it is useful to learn the statistical properties of MR to have a comprehensive understanding of the angle measurement performance. Thus, the statistical properties of MR were studied in many literatures [10-13]. In the literatures [10, 11], Seifer found an ingenious way to quantify the angular accuracy by calculating the mean and covariance of the real part of the MR in condition that the sum channel amplitude exceeds a detection threshold. Nonfluctuating and fluctuating target cases were considered and compared. In [12], Groves obtained the joint distribution of the real and imaginary parts of the MR with arbitrarily correlated Gaussian noise. Using the general result, the thresholding was considered and the marginal density function of the real part of the MR was derived for the case of real correlation between the channels. In order to obtain more general statistical properties, in [13], Chaumette presented the statistical prediction of monopulse 
angle measurement where the target fluctuation law was unknown and multiple observations were available. And the author successfully derived simple expression of conditional mean and variance of the MR for the case that only a single observation was required and the threshold detection was achieved.

The above research results can effectively describe the statistical properties of MR in many cases. They are very instructive for radar designers. Those researches mainly discussed the narrowband monopulse radar and assumed that the target was an ideal point target. Only the effects of Gaussian noise on the MR were considered. However, when the wideband monopulse radar is applied to track the complex targets on the sea, the target returns are spread within different range resolution cells. Other different angle measurement methods now need to extract the target angular information. Meanwhile, the presence of sea clutter will influence the angle measurement performance. In such cases, the above research results are no longer appropriate to describe the statistical properties of MR of wideband monopulse radar.

Fortunately, the clutter returns for high resolution radars can be well modeled by the compound Gaussian model which consists of the product of two components called "texture" and "speckle." In this compound Gaussian model, the local mean level of the speckle component is determined by the texture component. When the local mean level is given, the clutter can be regarded as the Gaussian distribution. The similar procedure presented by Seifer in literatures $[10$, 11] can now be applied to obtain the local distribution of MR. This local distribution of MR is then averaged over all possible values of the local mean level to compute the overall distribution of MR. Encouraged by this, we focus on the effects of sea clutter on angle measurement performance of wideband monopulse radar.

In the wideband monopulse radar system, the high resolution range profile (HRRP) of sum channel is firstly applied to accomplish the extended target detection and the positions of the range cells of the target are determined in the HRRP. Then, the corresponding HRRP returns in the sum and the difference channels are utilized to extract the angle of every one of the range cells of the target till the angles of all range cells are obtained. Lastly, the geometrical center angle of the target can be calculated by smoothing all angles. According to this procedure, the method presented in [10, 11] is developed to deduce the mathematical expression of conditional pdf of MR error for Rayleigh fluctuating extended target in the background of compound $K$-distributed sea clutter plus noise. Using this conditional pdf, the MR conditional root mean square error (RMSE), the conditional mean, and standard deviation (STD) of the MR error are then obtained to evaluate the angle measurement performance. Specifically, considering that the clutter may exhibit the correlation characteristics in the wideband monopulse radar, the spatial correlation of the texture components for each channel clutter and the correlation of the texture components between the sum and difference channel clutters are considered in discussion, and two extreme situations are tackled for each of them.
The rest of the paper is organized as follows: the basics of statistical analysis, including the radar returns model, the compound $K$-distribution model, and the binary detector, are introduced in Section 2. In Section 3, we deduce the conditional pdf of MR error, when the target is submerged in the compound $K$-distributed sea clutter and noise. Through numerical simulation method, effects of binary detector's parameters and $K$-distributed clutter on the angle measurement performance are analyzed in Section 4. Finally, Section 5 concludes the paper.

\section{Basics of Statistical Analysis}

2.1. Wideband Monopulse Radar Returns. The radar returns in the sum and difference channels can be, respectively, expressed as

$$
\begin{aligned}
& \widetilde{s}_{m}=s_{m}+c_{s m}+n_{s m}, \\
& \widetilde{d}_{m}=d_{m}+c_{d m}+n_{d m}, \\
& \quad m=1, \ldots, H,
\end{aligned}
$$

where $H$ is the number of the range cells of the target, $s_{m}$ and $d_{m}$ are the target returns in the sum and difference channels, respectively, when the clutter and noise are absent, $c_{s m}$ and $c_{d m}$ are the clutter components in the sum and difference channels, respectively, and $n_{s m}$ and $n_{d m}$ are the noise components in the sum and difference channels, respectively. In this paper, the noise components are the complex Gaussian noises with zero mean and variance $\sigma_{n s}^{2}$ for sum channel and $\sigma_{n d}^{2}$ for difference channel. The target returns, clutter, and noise are assumed to be statistically independent.

A variety of theoretical distribution functions (Rayleigh, Weibull, $K$, Pareto, etc.) were proposed to model the pdf of the amplitude of non-Gaussian sea clutter [14-16]. Among these distributions the compound $K$-distribution is widely used to model the clutter and many existing contributions analyzed the radar performance considering the compound $K$-distributed clutter [17-20]. For the sea clutter both in the sum and difference channels, the compound $K$-distribution can not only depict the amplitude distribution accurately but also explain the scattering mechanism of sea clutter well. According to the compound $K$-distribution, the clutter can be interpreted as the product of two independent components, such that

$$
c=y x
$$

where $x$ is the speckle and $y$ is the texture which is nonnegative random process. The mean level of the speckle component is modulated by the texture component. The texture and speckle are considered to be independent processes. Therefore, the pdf of compound $K$ distributed sea clutter can be expressed as

$$
p(|c|)=\int_{0}^{\infty} p(|c| \mid y) p(y) d y
$$


where $|c|$ is the amplitude of the sea clutter. $p(|c| \mid y)$ is the pdf of the speckle component and can be given by

$$
p(|c| \mid y)=\frac{2|c|}{y^{2} \sigma_{c}^{2}} \exp \left(-\frac{|c|^{2}}{y^{2} \sigma_{c}^{2}}\right)
$$

where $\sigma_{c}^{2}=P_{c}$ and $P_{c}$ is the average power of sea clutter. The pdf of $y$ can be written as

$$
p(y)=\frac{2 v^{v}}{\Gamma(v)} y^{2 v-1} \exp \left(-v y^{2}\right),
$$

where $\Gamma(\cdot)$ is Gamma function and $v$ is the clutter shape parameter which embodies the clutter fluctuation degree. Thus, the clutter in the sum and difference channels are expressed as $c_{s m}=y_{s m} x_{s m}$ and $c_{d m}=y_{d m} x_{d m}$, respectively. And the clutter average powers are denoted as $\sigma_{c s}^{2}$ and $\sigma_{c d}^{2}$ and the shape parameters are denoted as $v_{c s}$ and $v_{c d}$.

It is assumed that the target returns in all resolution cells are randomly fluctuating and follow the same distribution. Their amplitude pdfs are Rayleigh distributions and their phase pdfs are uniform distributions. So, $s_{m}$ is a complex Gaussian random variable with zero mean. Its average power is $\bar{A}_{m}^{2}$. We assume that, $\forall m, n \in[1, H], \bar{A}_{m}^{2}=\bar{A}_{n}^{2}=\bar{A}^{2}$. Thus, the target SCNR in the sum channel can be written as

$$
\chi_{\mathrm{SCNR}}=\frac{E\left\{\left|s_{m}\right|^{2}\right\}}{E\left\{\left|c_{s m}+n_{s m}\right|^{2}\right\}}=\frac{\bar{A}^{2}}{\sigma_{c s}^{2}+\sigma_{n s}^{2}},
$$

where $E\{\cdot\}$ denotes expectation operator. Additionally, the target conditional SCNR in the sum channel can be rewritten as

$$
\begin{aligned}
\chi_{2} & =\frac{E\left\{\left|s_{m}\right|^{2} \mid y_{s m}\right\}}{E\left\{\left|c_{s m}+n_{s m}\right|^{2} \mid y_{s m}\right\}}=\frac{\bar{A}^{2}}{y_{s m}^{2} \sigma_{c s}^{2}+\sigma_{n s}^{2}} \\
& =\frac{\sigma_{c s}^{2}+\sigma_{n s}^{2}}{y_{s m}^{2} \sigma_{c s}^{2}+\sigma_{n s}^{2}} \chi_{\mathrm{SCNR}},
\end{aligned}
$$

where $y_{s m}$ is the clutter texture in the sum channel.

2.2. Binary Detector. In the wideband radar system, the binary detector is employed for the effective detection of an extended target in sea clutter, because it can significantly decrease the false alarm probability induced by the sea spikes [21-24]. The binary detector is comprised of two stages. The first stage is essentially a simplified version of the likelihood ratio test (LRT) where a detection threshold $T$ is set according to the quiescent clutter and noise levels. For the $m$ th range cell, the detector output is set to either 1 or 0 according to

$$
\left|\widetilde{s}_{m}\right| \gtreqless T \longmapsto h_{m}^{(1)} .
$$

During this stage, all range cell returns are quantified as 1 or 0 . The second stage detection operates upon these binarydetected range returns and a range sliding window $\mathrm{N}$-of$P$ detector is used. Suppose that $K$ range cell returns are sampled. We denote that $j \in\{0,1, \ldots, K-P+1\}$ and $M=$ $\sum_{m=j}^{j+P-1} h_{m}^{(1)}$. Then, this stage is accomplished as

$$
M \gtreqless N \longmapsto h_{m}^{(2)} .
$$

If $M \geq N$, this indicates the presence of a target in this range sliding window, and thus $h_{m}^{(2)}=1$; otherwise this indicates that the target is not present, and thus $h_{m}^{(2)}=0$. Generally, the length of range sliding window is not shorter than the size of target.

For the high resolution radar, the sizes of range cells are smaller than the scales of large features on the sea surface. Therefore, the correlation between the sea clutter from adjacent range cells, that is, the spatial correlation, is generally more pronounced. Modeling of the spatial correlation characteristics is very important for accurate performance prediction of binary detector. For the compound $K$ distribution, the spatial correlation can be characterized by the correlation properties of the texture and speckle. Due to the absence of oversampling, the speckle can be considered decorrelated from one range cell to the next. Here, only two bounding cases of spatial correlation for the texture are discussed.

As given in Table 1 by Watts [25], the spatial correlation length of clutter in the different sea states is several to dozens of meters. When the length of range sliding window is much larger than the spatial correlation length of clutter, it can be assumed that the texture samples are independent within the range sliding window. This case is denoted as the spatially independent texture case. On the contrary, when the length of range sliding window is smaller than the spatial correlation length of clutter, it can be assumed that the texture samples are completely correlated in the range sliding window. This case is denoted as the spatially correlated texture case.

For the spatially independent texture case, the false alarm probability of the first stage detection, denoted as $P_{f 1}$, can be expressed as

$$
\begin{aligned}
P_{f 1} & =\int_{T}^{\infty} \int_{0}^{\infty} p\left(\left|c_{s m}\right| \mid y_{s m}\right) p\left(y_{s m}\right) d y_{s m} d\left|c_{s m}\right| \\
& =\int_{0}^{\infty} \exp \left(-\frac{|T|^{2}}{y_{s m}^{2} \sigma_{c s}^{2}+\sigma_{n s}^{2}}\right) p\left(y_{s m}\right) d y_{s m},
\end{aligned}
$$

where $p\left(y_{s m}\right)$ is the pdf of $y_{s m}$ given by (5). Thus, the overall false alarm probability for the binary detector can be computed by summing over the false alarm occurrences of the binomial pdf as

$$
P_{F 1}=\sum_{m=N}^{P} C_{m}^{P} P_{f 1}^{m}\left(1-P_{f 1}\right)^{P-m},
$$

where $C_{m}^{P}$ is the number of combinations of $P$ items taken $m$ at a time. The detection probability of the first stage detection can be obtained as

$$
\begin{aligned}
& P_{d 1}=p_{d}\left\{\left|\widetilde{s}_{m}\right| \geq T\right\} \\
& \quad=\int_{0}^{\infty} \exp \left(-\frac{|T|^{2}}{\left(1+\chi_{2}\right)\left(y_{s m}^{2} \sigma_{c s}^{2}+\sigma_{n s}^{2}\right)}\right) p\left(y_{s m}\right) d y_{s m} .
\end{aligned}
$$


Similar to the computation of the overall false alarm probability, the overall detection probability for the binary detector can be expressed as

$$
P_{D 1}=p_{d}\{M \geq N\}=\sum_{m=N}^{P} C_{m}^{P} P_{d 1}^{m}\left(1-P_{d 1}\right)^{P-m} .
$$

However, for the spatially correlated texture case, $y_{s m}$ is constant within the range sliding window but varies evidently with range sliding window locations. Hence, the calculating methods of the overall false alarm probability and the overall detection probability in this case are different from those in the independent texture case. For the first stage detection, given the local mean level value $y_{s m}$, the local false alarm probability $P_{f 2}$ and detection probability $P_{d 2}$ can be computed as

$$
\begin{aligned}
P_{f 2} & =\int_{T}^{\infty} p\left(\left|c_{s} m\right| \mid y_{s m}\right) d\left|c_{s} m\right| \\
& =\exp \left(-\frac{|T|^{2}}{y_{s m}^{2} \sigma_{c s}^{2}+\sigma_{n s}^{2}}\right), \\
P_{d 2} & =p_{d}\left\{\left|\widetilde{s}_{m}\right| \geq T \mid y_{s m}\right\} \\
& =\exp \left(-\frac{|T|^{2}}{\left(1+\chi_{2}\right)\left(y_{s m}^{2} \sigma_{c s}^{2}+\sigma_{n s}^{2}\right)}\right) .
\end{aligned}
$$

Then the overall probability of false alarm and detection for the binary detector can be computed by summing over the local probabilities of false alarm and detection of the binomial pdf and then by averaging over all possible values of the local mean level. They are given by

$$
\begin{aligned}
P_{F 2} & =\int_{0}^{\infty} \sum_{m=N}^{P} C_{m}^{P} P_{f 2}^{m}\left(1-P_{f 2}\right)^{P-m} p\left(y_{s m}\right) d y_{s m}, \\
P_{D 2} & =p_{d}\{M \geq N\} \\
& =\int_{0}^{\infty} \sum_{m=N}^{P} C_{m}^{P} P_{d 2}^{m}\left(1-P_{d 2}\right)^{P-m} p\left(y_{s m}\right) d y_{s m} .
\end{aligned}
$$

Once the overall false alarm probability and the parameters of binary detector are given, the detection threshold of the LRT in the first stage detection can be determined by (10) and (11) for the spatially independent texture case and by (14) and (16) for the spatially correlated texture case. Then, the aggregated detection probabilities in both these two cases can be computed.

\section{Angle Measurement Performance of Wideband Monopulse Radar}

3.1. Angle Measurement Error of Wideband Monopulse Radar. Without loss of generality, we do not consider the implementation type of the monopulse radar. In a practical environment, the received radar signal usually contains clutter, noise, and interference. The MR is a complex number. In real applications, the real part of the complex MR(CMR) is widely used to extract the angle of arrival (DOA) of a target, which is defined as $[1,10]$

$$
r=\operatorname{Re}\left\{\frac{d}{s}\right\}
$$

where $s$ and $d$, respectively, are the complex-valued samples of receiving signals simultaneously obtained from the sum and difference channels. $\operatorname{Re}\{\cdot\}$ denotes the real part of a complex value. $r$ is the monotone function of DOA $\theta$ of the observed target. Usually, the relationship between $r$ and $\theta$ can be approximated by a linear function; namely,

$$
\theta \approx \frac{\theta_{\mathrm{BW}}}{k_{m}} r
$$

where $\theta_{\mathrm{BW}}$ is the $3 \mathrm{~dB}$ beamwidth of the sum pattern and $k_{m}$ denotes monopulse slope.

We assume that $M$ range cell returns are determined to have the probable presence of a target and the presence of a target is determined by the detection process. According to (18) and (19), we can obtain the angle value $\theta_{m}$ for each range cell of target. The geometrical center angle of target is then acquired by weighting processing on all angle values; that is,

$$
\theta=\sum_{m=1}^{M} \alpha_{m} \theta_{m}
$$

where $\alpha_{m}$ is weighting operator. The commonly used weighting operators include uniform weighting, linear weighting, and power weighting. To simplify the analysis, the uniform weighting method is adopted; that is, $\alpha_{m}=1 / M$.

When clutter and noise are absent, the MR in the $m$ th range cell is denoted as $\eta_{m}=d_{m} / s_{m}$. It is a real number. The monopulse slope $k_{m}$ is assumed to be constant for all range cell returns from the same target. Thus, according to (20), the $\mathrm{MR}$ associated with the real geometrical center angle of target can be written as

$$
\eta=\frac{1}{M} \sum_{m=1}^{M} \eta_{m}=\frac{1}{M} \sum_{m=1}^{M} \frac{d_{m}}{s_{m}} .
$$

The flare angle of target and the corresponding MR are denoted as $\Delta \theta$ and $\Delta \eta$, respectively. $\Delta \theta$ is related to the target size and the radar working range. When radar works in the far field, we can use the approximations $\Delta \theta \approx 0$ and $\eta_{m} \approx \eta$. Here, the angle measurement error induced by the flare angle of target is ignored. When the target return is submerged in the sea clutter and noise, the CMR of target return of single range cell is denoted as

$$
\tilde{\eta}_{m}=\frac{\widetilde{d}_{m}}{\widetilde{s}_{m}}=\frac{d_{m}+c_{d m}+n_{d m}}{s_{m}+c_{s m}+n_{s m}} .
$$

Then, the CMR error of target can be expressed as

$$
\begin{aligned}
\varepsilon & =\frac{1}{M} \sum_{m=1}^{M} \tilde{\eta}_{m}-\frac{1}{M} \sum_{m=1}^{M} \eta_{m}=\frac{1}{M} \sum_{m=1}^{M}\left(\frac{\tilde{d}_{m}}{\widetilde{s}_{m}}-\frac{d_{m}}{s_{m}}\right) \\
& =\frac{1}{M} \sum_{m=1}^{M} \varepsilon_{m},
\end{aligned}
$$


where $\varepsilon_{m}$ is the CMR error of single range cell. By using $\eta_{m} \approx$ $\eta, \varepsilon_{m}$ can be expressed as

$$
\varepsilon_{m}=\frac{\widetilde{d}_{m}}{\widetilde{s}_{m}}-\frac{d_{m}}{s_{m}} \approx \frac{\left(c_{d m}+n_{d m}\right)-\eta\left(c_{s m}+n_{s m}\right)}{s_{m}+c_{s m}+n_{s m}} .
$$

The denominator in (24) is denoted as $S_{m}=s_{m}+c_{s m}+n_{s m}$ and the numerator is denoted as $D_{m}=\left(c_{d m}+n_{d m}\right)-\eta\left(c_{s m}+n_{s m}\right)$. Here, $D_{m}$ represents the error sources of angle measurement, including the clutter and noise in both the sum and difference channels. Moreover, the contribution of $\left(c_{d m}+n_{d m}\right)$ to the error is given by $\left(c_{d m}+n_{d m}\right) /\left(s_{m}+c_{s m}+n_{d m}\right)$ and the contribution of $\left(c_{s m}+n_{s m}\right)$ to the error is given by $\eta\left(c_{s m}+\right.$ $\left.n_{s m}\right) /\left(s_{m}+c_{s m}+n_{d m}\right)$. The effect of the clutter plus noise of sum channel is related to the location of target in the beam. Then, we can get $E\left[S_{m}\right]=0$ and $E\left[D_{m}\right]=0$. The conditional variances of $S_{m}$ and $D_{m}$ can be represented as

$$
\begin{aligned}
a & =\sqrt{\frac{1}{2}\left(E\left[\left|D_{m}-E\left[D_{m}\right]\right|^{2} \mid y_{s m}, y_{d m}\right]\right)} \\
& =\sqrt{\frac{1}{2}\left[y_{d m}^{2} \sigma_{c d}^{2}+\sigma_{n d}^{2}+\eta^{2}\left(y_{s m}^{2} \sigma_{c s}^{2}+\sigma_{n s}^{2}\right)-2 \rho_{1} \eta y_{s m} \sigma_{c s} y_{d m} \sigma_{c d}\right]},
\end{aligned}
$$

$$
\begin{aligned}
b & =\sqrt{\frac{1}{2}\left(E\left[\left|S_{m}-E\left[S_{m}\right]\right|^{2} \mid y_{s m}, y_{d m}\right]\right)} \\
& =\sqrt{\frac{1}{2} E\left\{\left|s_{m}+c_{s m}+n_{s m}\right|^{2}\right\}}=\sqrt{\frac{1}{2}\left(1+\chi_{2}\right)\left(y_{s m}^{2} \sigma_{c s}^{2}+\sigma_{n s}^{2}\right)},
\end{aligned}
$$

where $\rho_{1}$ is the real part of conditional correlation of clutter between the sum and the difference channels. This conditional correlation can be represented as

$$
\begin{aligned}
\xi_{1} & =\rho_{1}+j \varsigma_{1}=\frac{E\left\{c_{s m}^{*} c_{d m} \mid y_{s m}, y_{d m}\right\}}{\sqrt{E\left\{c_{s m}^{*} c_{s m} \mid y_{s m}\right\}} \sqrt{E\left\{c_{d m}^{*} c_{d m} \mid y_{d m}\right\}}} \\
& =\frac{E\left\{y_{s m} y_{d m} x_{s m}^{*} x_{d m} \mid y_{s m}, y_{d m}\right\}}{y_{s m} y_{d m} \sigma_{c s} \sigma_{c d}}=\frac{E\left\{x_{s m}^{*} x_{d m}\right\}}{\sigma_{c s} \sigma_{c d}} \\
& =\xi_{x},
\end{aligned}
$$

where $\varsigma_{1}$ is imaginary part of $\xi_{1} . \rho_{1}$ and $\varsigma_{1}$ are bounded by $\rho_{1}^{2}+\varsigma_{1}^{2} \leq 1 . \xi_{x}$ is the correlation between the speckles of the sum and the difference channels. The superscript $*$ denotes the conjugate operation. From (26), we observe that the conditional correlation $\xi_{1}$ is equivalent to the speckle correlation $\xi_{x}$. Then, the conditional correlation between $S_{m}$ and $D_{m}$ is given by

$$
\begin{aligned}
\xi_{\varepsilon} & =\frac{E\left\{\left(s_{m}+c_{s m}+n_{s m}\right)^{*}\left[\left(c_{d m}+n_{d_{m}}\right)-\eta\left(c_{s m}+n_{s m}\right)\right] \mid y_{s m}, y_{d m}\right\}}{2 a b}=\frac{y_{s m} \sigma_{c s}}{2 a b}\left(\rho_{1} y_{d m} \sigma_{c d}-\eta y_{s m} \sigma_{c s}\right) \\
& +j \frac{y_{s m} y_{d m} \sigma_{c s} \sigma_{c d}}{2 a b} \varsigma_{1}=\rho_{\varepsilon}+j \varsigma_{\varepsilon},
\end{aligned}
$$

where $\rho_{\varepsilon}$ and $\varsigma_{\varepsilon}$ are the real and imaginary parts of $\xi_{\varepsilon}$, respectively, and are bounded by $\rho_{\varepsilon}^{2}+\varsigma_{\varepsilon}^{2} \leq 1$. It should be noted that $\rho_{\varepsilon}$ and $\varsigma_{\varepsilon}$ have $y_{s m}$ and $y_{d m}$ dependencies, but they are constant in the Gaussian noise case.

\subsection{Exact Solution for Conditional pdf of MR Error. Let us} define

$$
L_{m}=\frac{\left|\widetilde{s}_{m}\right|}{\sqrt{E\left[\left|c_{s m}+n_{s m}\right|^{2} \mid y_{s m}\right]}} .
$$

Denote $Q_{\varepsilon m}=D_{m} / S_{m}$. The CMR error $q_{\varepsilon}$ can be represented by the terms of its real and imaginary parts; namely, $q_{\varepsilon m}=$ $r_{\varepsilon m}+j t_{\varepsilon m}$. Thus, the joint pdf of $Q_{\varepsilon m}$ and $L_{m}$ can be represented as follows [10]:

$$
\begin{aligned}
& p_{R \varepsilon m \mid \Delta_{1}}\left(r_{\varepsilon m} \mid y_{s m}, y_{d m}\right)=\frac{2 l_{m}^{3}}{\pi \mu^{2}\left(1+\chi_{2}\right)^{2}} \\
& \quad \times \exp \left(-\left[\frac{l_{m}^{2}}{\left(1+\chi_{2}\right)}+\frac{l_{m}^{2}}{\left(1+\chi_{2}\right) \mu^{2}}\left(r_{\varepsilon m}-\frac{a}{b} \rho_{\varepsilon}\right)^{2}\right.\right. \\
& \left.\left.+\frac{l_{m}^{2}}{\left(1+\chi_{2}\right) \mu^{2}}\left(t_{\varepsilon m}-\frac{a}{b} \varsigma_{\varepsilon}\right)^{2}\right]\right),
\end{aligned}
$$

where $\chi_{2}, \rho_{\varepsilon}, \varsigma_{\varepsilon}, a$, and $b$ are defined previously, and

$$
\mu=\frac{a}{b} \sqrt{1-\rho_{\varepsilon}^{2}-\varsigma_{\varepsilon}^{2}}
$$

It should be noted that the variation of the underlying power due to the $K$-distribution assumption now causes the pdf in (29) to have $y_{s m}$ and $y_{d m}$ dependencies.

At the first stage of detection process, $\Delta_{1}=\left\{\left|\widetilde{s}_{m}\right| \geq T\right\}$ denotes the event that the radar return of single range cell in the sum channel exceeds the detection threshold. $l_{0}=$ $T / \sqrt{E\left[\left|c_{s m}+n_{s m}\right|^{2} \mid y_{s m}\right]}$ denotes the detection threshold of the LRT normalized with respect to the conditional rootmean-squared sum channel clutter plus noise power. It should be noted that the normalized values of $L_{m}$ and $l_{0}$ also have a $y_{s m}$ dependence under the $K$-distribution assumption. The resulting distribution conditioned on the occurrence of event $\Delta_{1}$ is then obtained by integrating with respect to variables $t_{\varepsilon m}$ and $l_{m}$ as follows:

$$
\begin{aligned}
& P_{R \varepsilon m \mid \Delta_{1}}\left(r_{\varepsilon m} \mid y_{s m}, y_{d m}, \Delta_{1}\right) \\
& =\frac{\mu^{2}}{P_{d} \sqrt{\pi}} B^{-3 / 2}
\end{aligned}
$$




$$
\begin{aligned}
& \times\left\{\frac{\sqrt{\pi}}{2}\left[1-\operatorname{erf}\left(\frac{B l_{0}}{\mu \sqrt{\left(1+\chi_{2}\right)}}\right)\right]\right. \\
& \left.+\frac{B l_{0}}{\mu \sqrt{\left(1+\chi_{2}\right)}} \exp \left[-\frac{B^{2} l_{0}^{2}}{\left(1+\chi_{2}\right) \mu^{2}}\right]\right\} \\
& =\frac{p_{R \varepsilon m}\left(r_{\varepsilon m}, \Delta_{1} \mid y_{s m}, y_{d m}\right)}{P_{d}}
\end{aligned}
$$

where $B=\mu^{2}+\left(r_{\varepsilon m}-(a / b) \rho_{\varepsilon}\right)^{2}, \operatorname{erf}(z)=(2 / \sqrt{\pi}) \int_{0}^{z} e^{-t^{2}} d t$ is error function, and $P_{d}=P\left\{\left|\widetilde{s}_{m}\right| \geq T \mid y_{s m}\right\}$ is the detection probability with the given $y_{s m}$ during the first stage detection.

Analogous to the effect on the detection performance, the spatial correlation of clutter also affects angle measurement performance of radar. We assume that the spatial correlation of the difference channel clutter is the same as the one of the sum channel clutter. In the spatially independent texture case, the value of $p_{R \varepsilon m \mid \Delta_{1}}\left(r_{\varepsilon m} \mid y_{s m}, y_{d m}, \Delta_{1}\right)$ is averaged over all possible values of $y_{s m}$ and $y_{d m}$ to compute the conditional pdf of MR error of single range cell return of target (denoted by $\left.p_{R \varepsilon m}\left(r_{\varepsilon m} \mid \Delta_{1}\right)\right)$; that is,

$$
\begin{aligned}
& p_{R \varepsilon m}\left(r_{\varepsilon m} \mid \Delta_{1}\right)=\frac{1}{P_{d 1}} \\
& \cdot \int_{0}^{\infty} \int_{0}^{\infty} p_{R \varepsilon m}\left(r_{\varepsilon m}, \Delta_{1} \mid y_{s m}, y_{d m}\right) \\
& \cdot p\left(y_{s m}, y_{d m}\right) d y_{s m} d y_{d m},
\end{aligned}
$$

where $P_{d 1}$ is the detection probability of the first stage detector in the spatially independent texture case and is given by (12). $p\left(y_{s m}, y_{d m}\right)$ is the joint pdf of $y_{s m}$ and $y_{d m}$.

It is assumed that $M$ range cell returns have been detected to determine the probable presence of a target in the local range region $[j, j+P-1)$ at the first stage of detection processing. Let $p\left(r_{\varepsilon} \mid M\right)$ denote the conditional pdf of MR error when $M$ range cell returns are used to extract the DOA of target. It can be assumed that for each range cell $p_{R \varepsilon m \mid \Delta_{1}}\left(r_{\varepsilon m}\right)$ is independent and obeys the distribution in (32). Denoting $r_{\varepsilon_{-} \text {sum }}=\sum_{m=1}^{M} r_{\varepsilon m}$ and $r_{\varepsilon}=r_{\varepsilon_{-} \text {sum }} / M$, the conditional pdf of $r_{\varepsilon_{-} \text {sum }}$ can be computed as [26]

$$
p_{\text {sum }}\left(r_{\varepsilon_{-} \text {sum }} \mid M\right)=\left\{p_{R \varepsilon 1}\left(r_{\varepsilon 1} \mid \Delta_{1}\right) \otimes p_{R \varepsilon 2}\left(r_{\varepsilon 2} \mid \Delta_{1}\right) \otimes \cdots \otimes p_{R \varepsilon M}\left(r_{\varepsilon M} \mid \Delta_{1}\right)\right\}
$$

where $\otimes$ is convolution operation. Thus, we can obtain $p\left(r_{\varepsilon} \mid\right.$ $M)=M p_{\text {sum }}\left(M r_{\varepsilon} \mid M\right)$. Additionally, the probability of $M$ times occurrences of the event $\Delta_{1}$ at the first stage detection processing can be written as $p_{d}\{M\}=C_{M}^{P} P_{d 1}^{M}\left(1-P_{d 1}\right)^{P-M}$. Then, we can obtain that

$$
p\left(r_{\varepsilon}, M\right)=C_{M}^{P} p\left(r_{\varepsilon} \mid M\right) P_{d 1}^{M}\left(1-P_{d 1}\right)^{P-M} .
$$

At the second stage of detection processing, $\Delta_{2}=\{M \geq$ $N\}$ is denoted as the event of the target presence. For the independent texture case, the pdf of MR error under the event $\Delta_{2}$ can then be expressed as

$$
\begin{aligned}
p_{R \varepsilon \mid \Delta_{2}}\left(r_{\varepsilon}\right) & =\frac{p\left(r_{\varepsilon}, M \geq N\right)}{p_{d}(M \geq N)} \\
& =\frac{\sum_{M=N}^{P} C_{M}^{P} p\left(r_{\varepsilon} \mid M\right) P_{d 1}^{M}\left(1-P_{d 1}\right)^{P-M}}{P_{D 1}},
\end{aligned}
$$

where $P_{D 1}$ is the overall detection probability in the spatially independent texture case and is given by (13).

In the spatially correlated texture case, the derivation process of the conditional pdf of MR error is similar to that in the spatially independent texture case. The analogous formulas with (33), (34), and (35) can be obtained by the simple substitutions of $P_{d 2}$ and $p_{R \varepsilon m \mid \Delta_{1}}\left(r_{\varepsilon m} \mid y_{s m}, y_{d m}, \Delta_{1}\right)$ for $P_{d 1}$ and $p_{R \varepsilon m}\left(r_{\varepsilon m} \mid \Delta_{1}\right)$, and they are denoted by $p\left(r_{\varepsilon} \mid\right.$ $\left.M, y_{s m}, y_{d m}\right), p\left(r_{\varepsilon}, M \mid y_{s m}, y_{d m}\right)$, and $p\left(r_{\varepsilon} \mid y_{s m}, y_{d m}\right)$. They have $y_{s m}$ and $y_{d m}$ dependencies. Therefore, the overall conditional pdf of MR error for the spatially correlated texture case is computed by averaging the MR errors over all possible values of $y_{s m}$ and $y_{d m}$; that is,

$$
\begin{gathered}
p_{R \varepsilon \mid \Delta_{2}}\left(r_{\varepsilon}\right)=\int_{0}^{\infty} \int_{0}^{\infty} p_{R \varepsilon \mid \Delta_{2}}\left(r_{\varepsilon} \mid y_{c s}, y_{c d}\right) \\
\cdot p\left(y_{c s}, y_{c d} \mid \Delta_{2}\right) d y_{c s} d y_{c d}=\frac{1}{P_{D 2}} \\
\cdot \int_{0}^{\infty} \int_{0}^{\infty} \sum_{M=N}^{P} C_{M}^{P} p\left(r_{\varepsilon} \mid M, y_{c s}, y_{c d}\right) \\
\cdot P_{d 2}^{M}\left(1-P_{d 2}\right)^{P-M} p\left(y_{c s}, y_{c d}\right) d y_{c s} d y_{c d}
\end{gathered}
$$

where $P_{d 2}$ and $P_{D 2}$ are the first and second stage detection probabilities in the spatially correlated texture case and are given by (15) and (17), respectively.

In the above analysis, the joint pdf $p\left(y_{s m}, y_{d m}\right)$ is involved. This pdf describes the distribution of the clutter textures of the sum and difference channels within the same range cell. Generally, the clutter returns in the sum and difference channels are from the scattering of the common illuminating sea surface region. Hence, the correlation of the texture components of the sea clutter between the sum and difference channels within the same range cell is also pronounced. Moreover, because the directions of the sum and difference pattern main lobes are not consistent and the clutter intensity is dominated by the antenna pattern gain, the texture correlations are different for different range cells. Herein, two bounding situations (i.e., $y_{s m}$ and $y_{d m}$ are independent and 
are completely correlated) are considered separately. When $y_{s m}$ and $y_{d m}$ are independent, $p\left(y_{s m}, y_{d m}\right)$ can be separated and can be rewritten as $p\left(y_{s m}, y_{d m}\right)=p\left(y_{s m}\right) p\left(y_{d m}\right)$. When $y_{s m}$ and $y_{d m}$ are completely correlated, the values of $y_{s m}$ and $y_{d m}$ within the same range cell are the same. All involving pdfs of monopulse angle measurement errors have only a $y_{s m}$ dependence. Therefore, the double integrals in (32) and (36) are reduced to the single integral.

We wish to determine the error in an estimate of target angle due to the clutter and noise. Using (35) and (36), the conditional moments of $r_{\varepsilon}$ are then given by

$$
E\left[r_{\varepsilon}^{n} \mid \Delta_{2}\right]=\int_{-\infty}^{\infty} r_{\varepsilon}^{n} p_{R \varepsilon \mid \Delta_{2}}\left(r_{\varepsilon}\right) d r_{\varepsilon}, \quad n=1,2, \ldots
$$

For $n$ is equal to unity and two, the first and second moments of $r_{\varepsilon}$ can be obtained by integrating (37), which are denoted as $E\left[r_{\varepsilon} \mid \Delta_{2}\right]$ and $E\left[r_{\varepsilon}^{2} \mid \Delta_{2}\right]$, respectively. Also, the first moment is named the mean error (bias). Although each of the clutter and noise variables has a distribution with zero mean value, the mean error is not zero and it cannot exceed the width of the main lobe. The square root of the second moment is named the RMSE and is denoted by $\Upsilon\left[r_{\varepsilon} \mid \Delta_{2}\right]$. In practice, the RMSE is desired to measure the error and it is widely used to assess the performance for various kinds of measurement results. It is the combined effect of the mean error and the STD from this mean error. The conditional STD of $r_{\varepsilon}$ is denoted as $\sigma\left[r_{\varepsilon} \mid \Delta_{2}\right]$ and it can be obtained from

$$
\sigma^{2}\left[r_{\varepsilon} \mid \Delta_{2}\right]=E\left[r_{\varepsilon}^{2} \mid \Delta_{2}\right]-\left(E\left[r_{\varepsilon} \mid \Delta_{2}\right]\right)^{2} .
$$

It is interesting to note that, in theory, this conditional STD of $r_{\varepsilon}$ is infinite when the condition of $\Delta_{2}$ is removed $[1,10]$. In actual practice, however, the conversion from monopulse ratio to angle is implemented as being within the main lobe and the target is also presumed to be within the main lobe. The conditional STD of $r_{\varepsilon}$ is finite and its maximum possible value is of the order of the beamwidth. Consequently, $\Upsilon\left[r_{\varepsilon} \mid\right.$ $\Delta_{2}$ ] is also finite and its maximum possible value is of the order of the beamwidth. In our discussion, $\Upsilon\left[\begin{array}{lll}r_{\varepsilon} & \Delta_{2}\end{array}\right]$ is applied to assess the performance of the angle measurement of wideband monopulse radar in clutter plus noise and $E\left[r_{\varepsilon} \mid\right.$ $\left.\Delta_{2}\right]$ and $\sigma\left[r_{\varepsilon} \mid \Delta_{2}\right]$ are applied to determine the main contributions of the clutter and noise to angle error.

\section{Simulation Results and Analysis}

4.1. Optimal Binary Detection. Good detection performance and angle measurement accuracy are two main goals of wideband monopulse radar design. Generally, only when the target appears in the detected result, the processing of monopulse angle measurement is carried out. Herein, the detection performance of the binary detector is firstly analyzed in the background of $K$-distributed sea clutter plus Gaussian noise. As in the researches [22, 27], the optimal parameter settings are defined as the values of the binary detector parameters which make the detector establish a given detection probability at a given false alarm probability with the least SCNR. It is assumed that the parameter $P$ of binary detector in our simulations is optimal. According to the results given in [22], it can be set to be equal to the target radial length. This optimal value of $P$ is denoted as $P_{\text {opt }}$.

The $K$-distributed sea clutters are generated by using the spherically invariant random process (SIRP) method. The 20,000 Monte Carlo experiments are conducted. In the simulation, the overall false alarm probability is set to $10^{-4}$ and the required detection thresholds of the LRT are computed theoretically by (10) and (11) for the spatially independent texture case and by (14) and (16) for the spatially correlated texture case. Those detection thresholds are then used to obtain the theoretical and simulated detection probabilities. The variations of theoretical and simulated detection probabilities with different values of binary detector parameter $N$ for two bounding cases are shown in Figure 1, where $N_{I}$ and $N_{C}$ are denoted as the values of $N$ for the spatially independent texture case and the spatially correlated texture case, respectively.

From Figure 1, we first observe that the simulated detection probability agrees well with the theoretical detection probability for both two cases. This demonstrates that equations (10)-(17) are correct. Second, we observe that, for fixed values of $v_{c s}$, in contrast with the spatially independent texture case, the detection probability of the spatially correlated texture case decreases. However, the deterioration degree decreases with the increase of $v_{c s}$. This can be explained intuitively that the overall false alarm rate is dominated by a localized area of high clutter in the spatially correlated texture case, but it is dominated by the occasional regions of high clutter in the spatially independent texture case. Consequently, in order to keep the same overall false alarm rate, the fixed first threshold of the binary detector in the spatially correlated texture case is required to set a higher value than that in the spatially independent texture case. Nevertheless, the bigger the values of $v_{c s}$ are, the closer are the fixed first thresholds of two cases. Thus, the detection probabilities of the spatially correlated texture case are close to those of the spatially independent texture case. Similar results were also obtained in [25].

As mentioned above, the value of $N$ is chosen as the optimal setting of the binary detector second threshold when the binary detector requires the least SCNR to establish a given detection probability $P_{D}=0.9$ at a given false alarm probability $P_{F}=10^{-4}$. Therefore, we can see from Figure 1(a) that $N_{I}=4$ is the optimal parameter setting for the spatially independent texture case, where $v_{c s}=1$. However, the value of the optimal binary detector parameter is $N_{\text {opt }}=5$ for this case by using the experiential formula in [22]. The reason for this is that our theoretical and simulated results are obtained in the background of $K$-distributed sea clutter plus noise, but the experiential formula is derived in the background of only the $K$-distributed sea clutter. Moreover, in the practical application, the optimal parameter can be selected within a reasonable range. In Figure 1(a), the performances of the binary detectors, of which the parameters are selected as $N_{I}=4$ and $N_{I}=5$, respectively, are very close when their detection probabilities satisfy $P_{d}>0.6$. Thus, it is accepted that $N_{I}=5$ is also the optimal parameter setting. When 


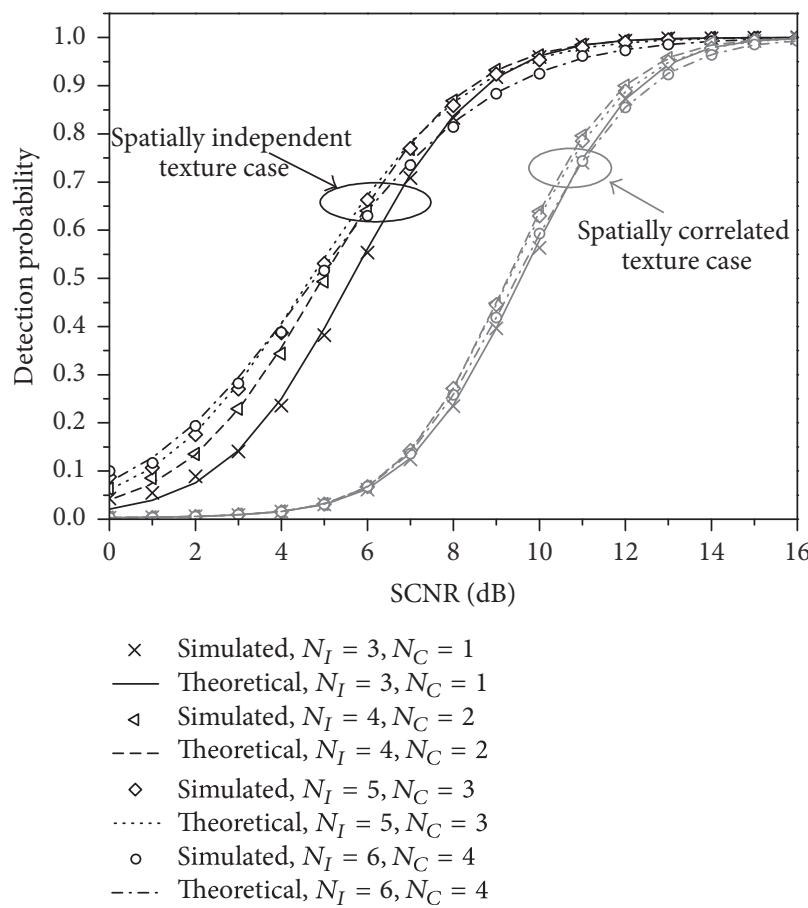

(a)

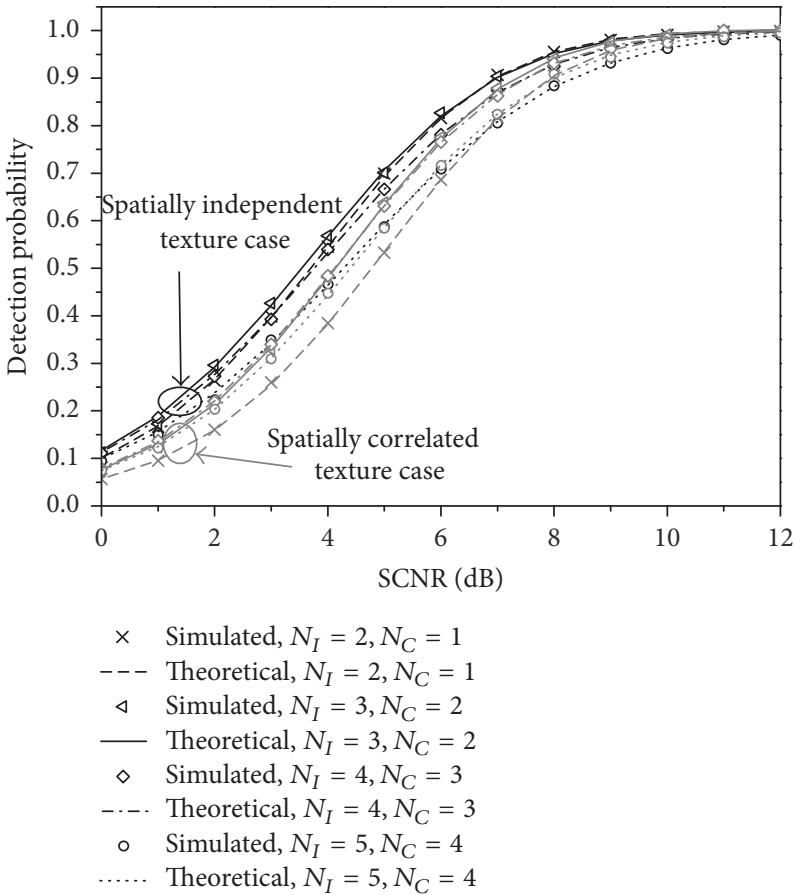

(b)

FIGURE 1: Theoretical and simulated radar detection probabilities for $\sigma_{n s}^{2}=1, P_{f}=10^{-4}, P_{\text {opt }}=8$, and CNR $=10 \mathrm{~dB}$ (black: spatially independent texture case, gray: spatially correlated texture case.). (a) $v_{c s}=1$; (b) $v_{c s}=25$.

$v_{c s}=25$, the optimal parameter setting is $N_{\mathrm{opt}}=3$ according to the experiential formula for the spatially independent texture case. We can see from Figure 1(b) that the detection performance of the detector is also optimal when $N_{I}=$ 3. Similarly, we can see from Figure 1 that $N_{C}=2$ and $N_{C}=3$ are the optimal settings of the binary detector second threshold for both $v_{c s}=1$ and $v_{c s}=25$ in the spatially correlated texture case. It seems that these results are not consistent with the known results in [27]. This is because in [27] only the $K$-distributed sea clutter was considered.

The above simulation demonstrates that the experiential formula given in [22] and the results given in [27] cannot be referred to in order to determinate the optimal parameter $N_{\text {opt }}$ of binary detector for the case that the radar returns include the $K$-distributed sea clutter and noise simultaneously. Although the spatial correlation of texture components affects the value of optimal second threshold setting of the binary detector, it should be noticed that, for large clutter shape parameter, the optimal parameter setting in the spatially correlated texture case is close to that in the spatially independent texture case. Moreover, the optimal parameter setting of $N$ in the spatially independent texture case is also the suboptimal parameter setting in the spatially correlated texture case for the small shape parameter, such as $N_{C}=4$, shown in Figure 1(a). Therefore, in the following investigation, the optimal parameter setting is discussed only for the spatially independent texture case.

In Figure 2, $N_{\text {opt }}$ is plotted against CNR for different shape parameters of sea clutter. As we can see from these curves,

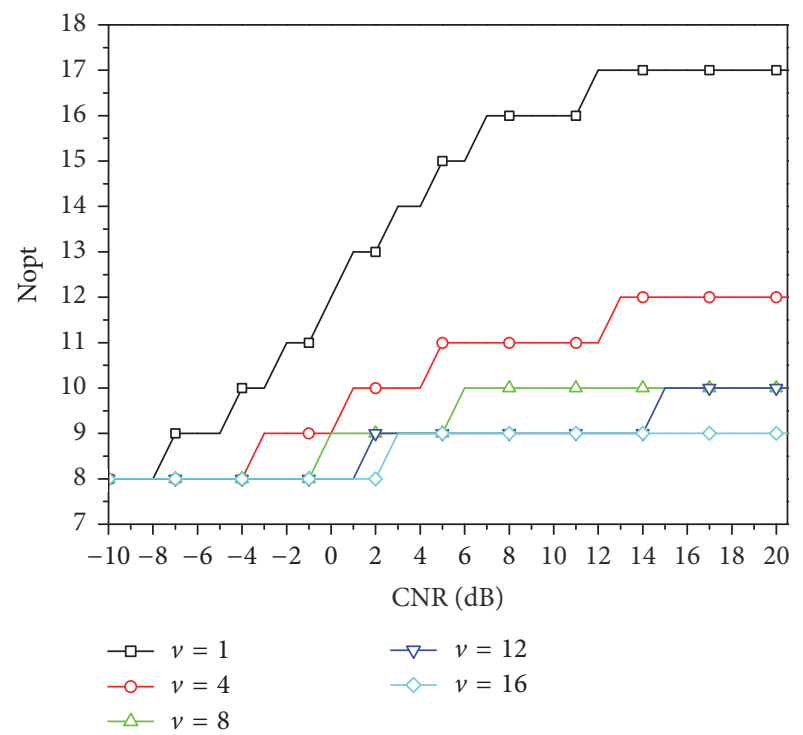

FIGURE 2: $N_{\text {opt }}$ against CNR in different shape parameters, for $\sigma_{n s}^{2}=$ $1, P_{f}=10^{-4}$, and $P_{\text {opt }}=30$.

$N_{\text {opt }}$ is a monotonously nondecreasing function of CNR. For each curve, there are three CNR regions, that is, the low CNR region (e.g., less than $-8 \mathrm{~dB}$ ), the middle CNR region (e.g., from $-8 \mathrm{~dB}$ to $15 \mathrm{~dB}$ ), and the high $\mathrm{CNR}$ region (e.g., greater than $15 \mathrm{~dB}$ ). In the middle $\mathrm{CNR}$ region, the value of $N_{\text {opt }}$ increases with the increase of CNR for the same clutter 
shape parameter. This is because the proportion of the effect of clutter on detection performance increases as the CNR increases. In other two CNR regions, the value of $N_{\text {opt }}$ is unrelated to the CNR. In the low CNR region, the effect of noise on detection performance is dominant and that of clutter can be ignored. So $N_{\text {opt }}$ is a constant for all cases. On the contrary, in the high CNR region, the effect of clutter on detection performance is dominant and that of noise can be ignored. Thus, the optimal parameter of binary detector can be selected by using the experiential formula given in
[22]. Based on above analysis, the experiential formula can be modified to guide the application of the binary detector when target submerges in the $K$-distributed sea clutter plus noise. The modified experiential formula is given as follows:

When $\mathrm{CNR}<-8 \mathrm{~dB}$,

$$
N_{\mathrm{opt}} \approx \operatorname{round}\left[0.27 P_{\mathrm{opt}}\right]
$$

When $-8 \mathrm{~dB} \leq \mathrm{CNR}<15 \mathrm{~dB}$,

$$
N_{\mathrm{opt}} \approx \begin{cases}\operatorname{round}\left[\frac{(\mathrm{CNR}+8)}{23} 0.65 v^{-0.25} P_{\mathrm{opt}}+\frac{(15-\mathrm{CNR})}{23} 0.27 P_{\mathrm{opt}}\right], & v<10 \\ \text { round }\left[\frac{(\mathrm{CNR}+8)}{23} 0.34 P_{\mathrm{opt}}+\frac{(15-\mathrm{CNR})}{23} 0.27 P_{\mathrm{opt}}\right], & v \geq 10\end{cases}
$$

When CNR > $15 \mathrm{~dB}$,

$$
N_{\mathrm{opt}} \approx \begin{cases}\operatorname{round}\left[0.65 v^{-0.25} P_{\mathrm{opt}}\right], & v<10, \\ \operatorname{round}\left[0.34 P_{\mathrm{opt}}\right], & v \geq 10,\end{cases}
$$

where round $[\cdot]$ denotes rounding operation. It should be stated that, under the constraint that the difference of detection performance is no more than $0.2 \mathrm{~dB}$, this modified experiential formula can be applied for different $P_{\text {opt }}$, and $N_{\text {opt }}$ can be selected within some ranges so that this formula is not unique. In addition, although this modified experiential formula is obtained under the condition that $P_{f}=10^{-4}$ and $P_{D}=0.9$, it can be confirmed that the validity conditions of this formula can be extended to $10^{-4} \geq P_{F} \geq 10^{-10}$ and $0.6 \leq P_{D} \leq 0.95$ according to the further numerical calculation.

4.2. Angle Measurement Performance. In this section, curves of $E\left[r_{\varepsilon} \mid \Delta_{2}\right], \sigma\left[r_{\varepsilon} \mid \Delta_{2}\right]$, and $\Upsilon\left[r_{\varepsilon} \mid \Delta_{2}\right]$ against relevant parameters are shown to analyze the angle measurement performance with wideband monopulse radar. Because the exact analytical formulas of $E\left[\begin{array}{l|l}r_{\varepsilon} & \Delta_{2}\end{array}\right], \sigma\left[\begin{array}{l|l}r_{\varepsilon} & \Delta_{2}\end{array}\right]$, and $\Upsilon\left[r_{\varepsilon} \mid \Delta_{2}\right]$ cannot be derived, all results in this section are obtained by numerical integration method. It is assumed that the angle measurements in the elevation and azimuth directions can be regarded to be independent of each other. Thus, the patterns of the sum and difference channels in either direction can be represented as two one-dimensional patterns. A simulated monopulse antenna model is used to evaluate the performance of angle measurement. The patterns in the sum and difference channels can be represented as [13]

$$
\begin{aligned}
& g_{\Sigma}(\theta)=\frac{\sqrt{4 \pi}}{\lambda} \sqrt{W}\left[\frac{\sin (h(\theta))}{h(\theta)}\right] \\
& g_{\Delta}(\theta)=\frac{\sqrt{4 \pi}}{\lambda} \sqrt{3 W}\left[\frac{\sin (h(\theta))}{h(\theta)^{2}}-\frac{\cos (h(\theta))}{h(\theta)}\right],
\end{aligned}
$$

where $h(\theta)=\pi(W / \lambda) \sin (\theta), \lambda$ is wavelength, $W$ is the dimension of the antenna, and $\theta$ is the deviation angle from boresight. We set $\lambda=8 \mathrm{~mm}$ and $W=32.2 \mathrm{~cm}$. Hence, the beamwidth is about 1.42 degrees. It is worth pointing out that, in off-axis measurement, the error of angle estimation in clutter increases as the deviation angle of off-axis target increases. Nevertheless, the results of the effect of clutter on the angle estimation error can exhibit the similar behavior no matter where the target locates in the main lobe. The analysis conclusion can be obtained from the results with a given deviation angle. Hence, in our simulations, the deviation angle of the off-axis target is set to 0.3 degrees.

4.2.1. Effect of the Binary Detector Parameter $N$. The choice of the binary detector parameter $N$ will affect not only the detection results but also the number of range cells applied to obtain the angle measurement. Accordingly, the different values of the binary detector parameter $N$ will bring the different angle measurement performances. In Figure 3, $\Upsilon\left[\begin{array}{lll}r_{\varepsilon} & \Delta_{2}\end{array}\right]$ is plotted against SCNR of sum channel for different values of $N$. Four different correlation cases are investigated. It is assumed that in the simulation the clutter shape parameters of sum and difference channels are the same.

It can be observed from Figures 3(a) and 3(b) that in the spatially independent texture case the performance of angle measurement can be improved significantly by increasing the values of $N$ when SCNR is low, yet its improvement slows down when SCNR is high. Instead there are small differences among the performances of angle measurement for different values of $N$ in the spatially correlated texture case. Comparing Figures 3(c) and 3(d) to Figures 3(a) and 3(b) we see that the values of $\Upsilon\left[r_{\varepsilon} \mid \Delta_{2}\right]$ in the spatially correlated texture case are higher than those in the spatially independent texture case for the same SCNR and $N$. This implies that an increase in spatial correlation of the texture components results in a degeneration of the angle measurement performance for the wideband monopulse radar. On the other hand, comparing Figures 3(b) and 3(d) to Figures 3(a) and 3(c), we do not see the significant differences of $\Upsilon\left[r_{\varepsilon} \mid \Delta_{2}\right]$ for different correlations of the clutter texture components between the 


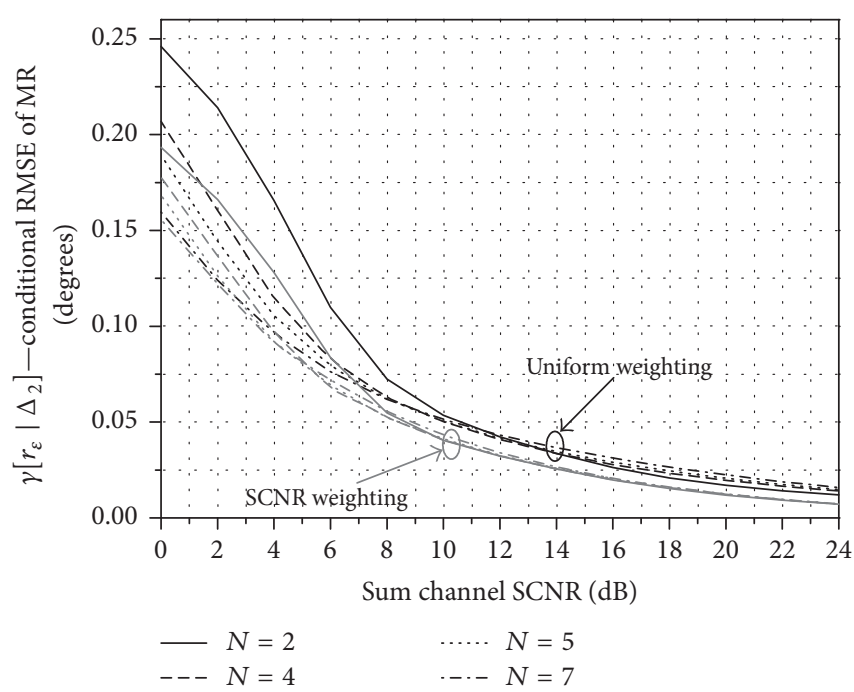

(a)

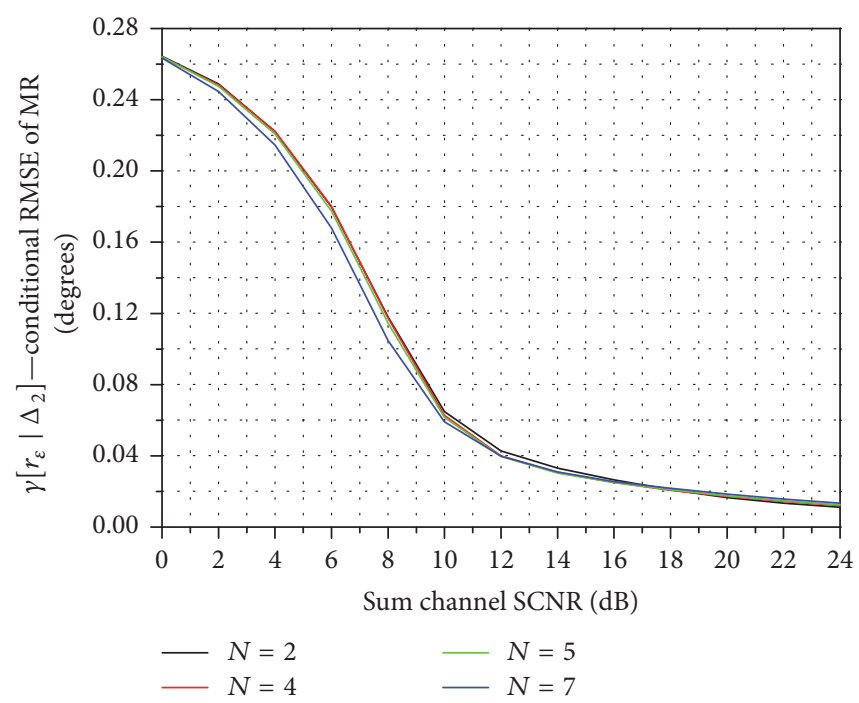

(c)

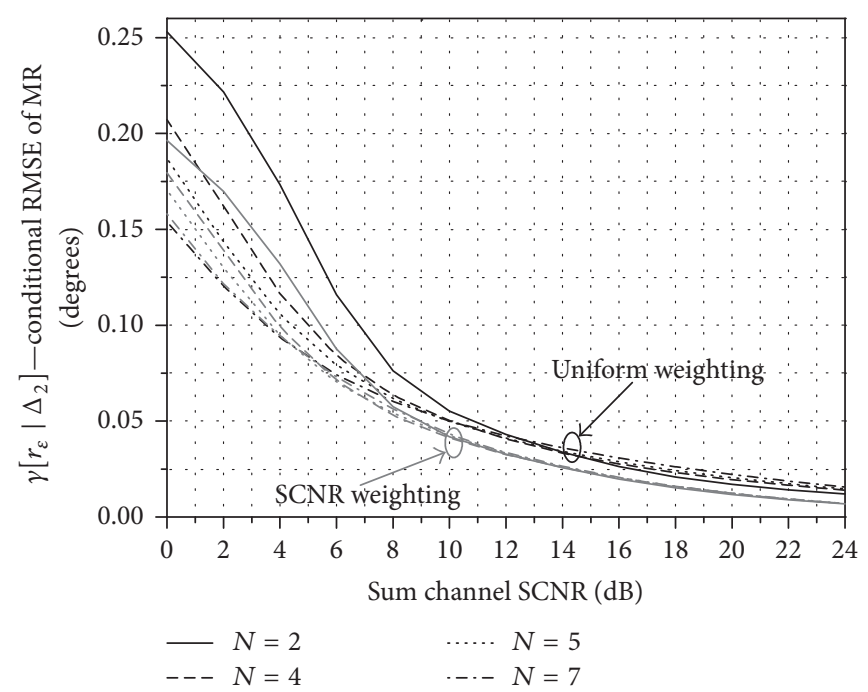

(b)

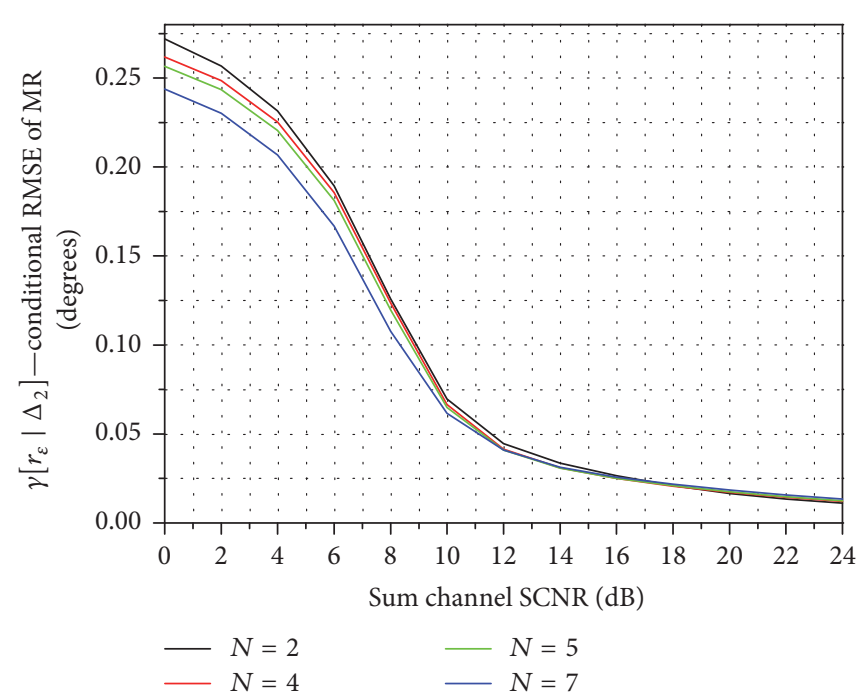

(d)

FIGURE 3: Conditional RMSE of MR in different values of $N$, for $\sigma_{n s}^{2}=1, P_{f}=10^{-4}, P_{\mathrm{opt}}=8, \theta=0.3$, and $v=1$; CNRs of sum and difference channels are $20 \mathrm{~dB}$ and $15 \mathrm{~dB}$, respectively. (a) Spatially independent texture case: $y_{s m}$ and $y_{d m}$ are independent. (b) Spatially independent texture case: $y_{s m}$ and $y_{d m}$ are completely correlated. (c) Spatially correlated texture case: $y_{s m}$ and $y_{d m}$ are independent. (d) Spatially correlated texture case: $y_{s m}$ and $y_{d m}$ are completely correlated.

sum and difference channels. This indicates that the effects of the correlation of the clutter texture components between the sum and difference channels on the angle measurement are very weak and can be ignored. According to the above results, in order to ensure the optimal radar detection performance and minimize the error of monopulse angle measurement, we can deduce a principle: the maximum among all values of $N$, which make the binary detector achieve the optimal detection performance, can be regarded as the optimal parameter setting.

Figure 4 shows the results of $E\left[r_{\varepsilon} \mid \Delta_{2}\right]$ and $\sigma\left[r_{\varepsilon} \mid \Delta_{2}\right]$ for different values of $N$ when the texture is independent in range and $y_{s m}$ and $y_{d m}$ are independent. It can be seen in Figure 4(a) that the absolute value of $E\left[\begin{array}{l|l}r_{\varepsilon} & \Delta_{2}\end{array}\right]$ decreases as the value of $N$ increases, and for different values of $N$ the differences among the curves of $E\left[\begin{array}{lll}r_{\varepsilon} & \Delta_{2}\end{array}\right]$ decrease as the SCNR increases. However, in Figure 4(b), the value of $\sigma\left[r_{\varepsilon} \mid \Delta_{2}\right]$ increases as the value of $N$ increases in the high SCNR region. For different values of $N$, the differences among the curves of $\sigma\left[\begin{array}{lll}r_{\varepsilon} & \Delta_{2}\end{array}\right]$ are small and decrease slowly as the SCNR increases. In addition, we can obtain by comparing Figures 3(a) and 4(a) that the value of $\Upsilon\left[r_{\varepsilon} \mid \Delta_{2}\right]$ approximates to the absolute value of $E\left[r_{\varepsilon} \mid \Delta_{2}\right]$ in the low SCNR region (e.g., less than $9 \mathrm{~dB}$ ). Similarly, we can obtain by comparing Figures 3(a) and 4(b) that the value of $\Upsilon\left[r_{\varepsilon} \mid \Delta_{2}\right]$ approximates to the value of $\sigma\left[r_{\varepsilon} \mid \Delta_{2}\right]$ in the high SCNR region (e.g., greater than $9 \mathrm{~dB}$ ). These phenomena indicate that the main source of the error in monopulse measurement 


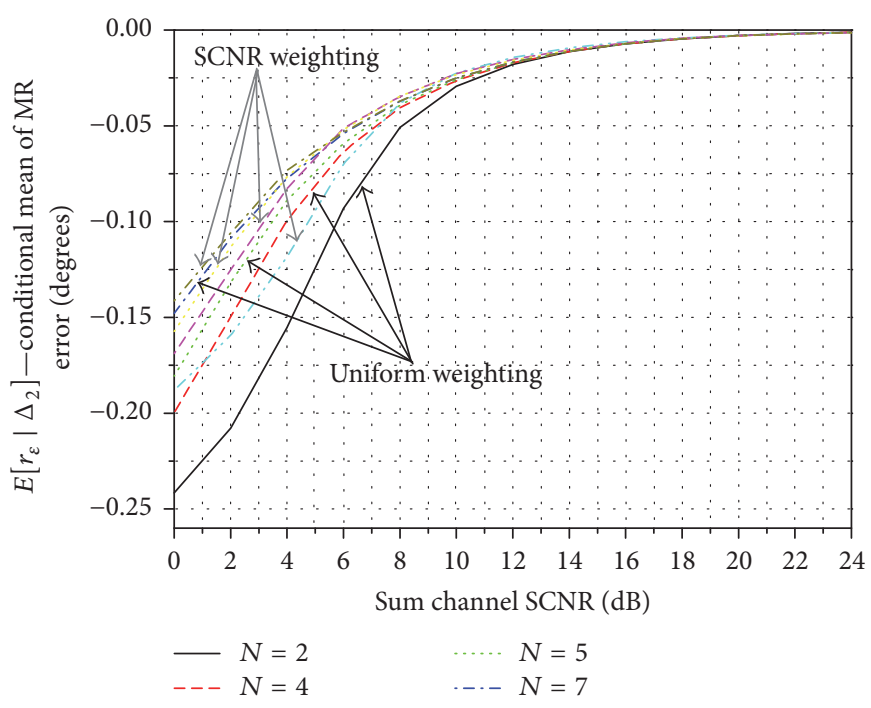

(a)

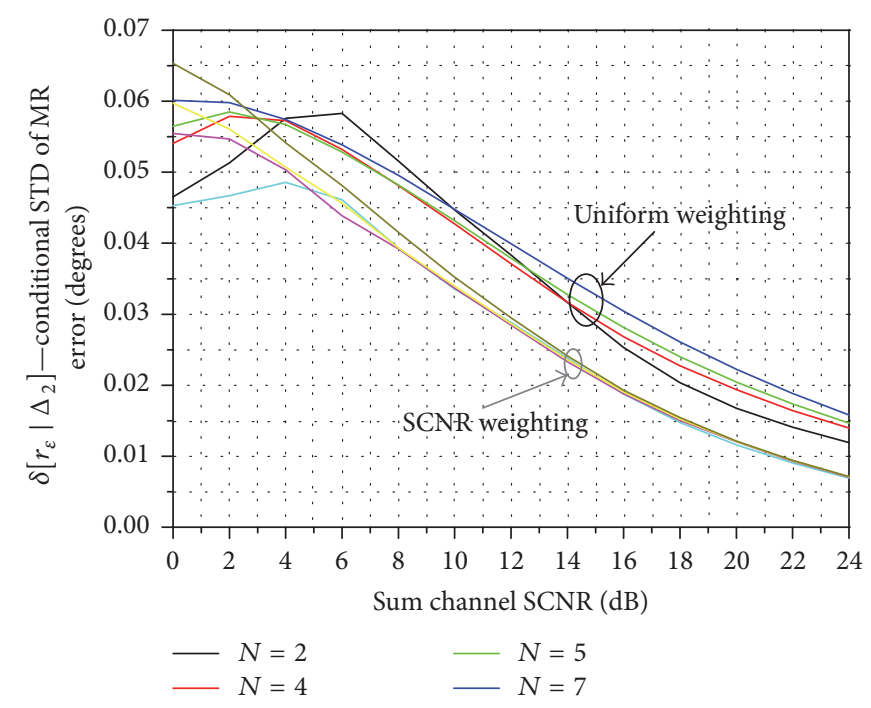

(b)

FIGURE 4: Conditional mean and conditional STD of MR error in different values of $N$ when the texture is independent in range and $y_{s m}$ and $y_{d m}$ are independent, for $\sigma_{n s}^{2}=1, P_{f}=10^{-4}, P_{\mathrm{opt}}=8, \theta=0.3$, and $v=1$; CNRs of sum and difference channels are $20 \mathrm{~dB}$ and $15 \mathrm{~dB}$, respectively. (a) Conditional mean of MR error; (b) conditional STD of MR error.

is the absolute bias for low SCNR and the standard deviation for high SCNR, respectively. Consequently, in Figure 3(a), $\Upsilon\left[r_{\varepsilon} \mid \Delta_{2}\right]$ decreases as the value of $N$ increases in the low SCNR region and increases in the high SCNR region.

The power weighting scheme is widely applied to estimate the angle from $N$ independent samples [28]. In Figures 3(a), $3(\mathrm{~b})$, and 4 , the results of $\Upsilon\left[r_{\varepsilon} \mid \Delta_{2}\right], E\left[r_{\varepsilon} \mid \Delta_{2}\right]$, and $\sigma\left[r_{\varepsilon} \mid \Delta_{2}\right]$ with respect to the power weighting scheme are also displayed to compare with those with respect to uniform weighting schemes. Visual inspection of these results demonstrates that the power weighting scheme can provide better angle measurement than the uniform weighting scheme in our simulation. Nevertheless, for these two weighting schemes, there are not very large differences in the angle measurement errors, and both the improvements of angle measurement performance with the increase of SCNR and the changes of angle measurement performance with the variation of $N$ show the similar tendencies. This means that either of weighting schemes is used to analyze the effect of the clutter plus noise on angle measurement of wideband monopulse radar; the same conclusion can be obtained. Therefore, in order to simplify the analysis, it can be accepted that the uniform weighting scheme is adopted in our analysis and simulation.

4.2.2. Effect of the Shape Parameter of Sea Clutter. It is well known that the shape parameter of sea clutter affects the detection performance of radar. Similarly, it also affects the angle measurement performance. It is necessary to develop the corresponding analysis to investigate this effect. In Figure 5, the results of $\Upsilon\left[r_{\varepsilon} \mid \Delta_{2}\right]$ are plotted against the SCNR of sum channel for different clutter shape parameters in four different correlation cases, and in Figure 6, the results of $E\left[r_{\varepsilon} \mid \Delta_{2}\right]$ and $\sigma\left[r_{\varepsilon} \mid \Delta_{2}\right]$ are plotted against the SCNR of sum channel for different clutter shape parameters in the case where the texture is independent in range and $y_{s m}$ and $y_{d m}$ are independent. In each simulation, the value of $N_{\text {opt }}$ is selected according to the above principle of the optimal parameter setting.

Figure 5 shows that, for low SCNR of sum channel, the angle measurement performance of wideband monopulse radar degrades with the decrease of the clutter shape parameter. The reason is that the clutter has a longer tail for the smaller shape parameter; hence, the target-like sea spike may have much higher power, which can cause the bigger bias between the measurement and real angles. This phenomenon can also be observed clearly in Figure 6(a). In addition, in Figure 5, $\Upsilon\left[\begin{array}{l|l}r_{\varepsilon} & \Delta_{2}\end{array}\right]$ decreases significantly as $v$ increases from 0.5 to 5 and decreases a little as $v$ increases from 5 to 20. This indicates that improvement of angle measurement performance slows down as $v$ increases. In Figures 5(a) and 6(b), we can see that both $\Upsilon\left[\begin{array}{l|l}r_{\varepsilon} & \Delta_{2}\end{array}\right]$ and $\sigma\left[\begin{array}{l|l}r_{\varepsilon} & \Delta_{2}\end{array}\right]$ do not decrease as $v$ increases in the high SCNR region. The reason is that $N_{\text {opt }}$ decreases as $v$ increases, but $\sigma\left[r_{\varepsilon} \mid\right.$ $\Delta_{2}$ ] decreases with the decrease of $N_{\text {opt }}$. In the high SCNR region, the decreasing degree of $\sigma\left[r_{\varepsilon} \mid \Delta_{2}\right]$ is greater than its increasing degree, and $\sigma\left[r_{\varepsilon} \mid \Delta_{2}\right]$ is the main error source of angle measurement. However, for different clutter shape parameters, there are not tremendous differences among the values of $\sigma\left[r_{\varepsilon} \mid \Delta_{2}\right]$.

In Figures 5(a) and 5(c), it should be noticed that as long as the shape parameters of sum channel clutter are the same, all results are very close for different clutter shape parameters of difference channel. Hence, in the preceding and following simulations, it is acceptable assumption that the clutter shape parameters of sum and difference channels are the same. In addition, the comparisons of Figures 5(a) and 5(b) with Figures 5(c) and 5(d) indicate that, for the same 

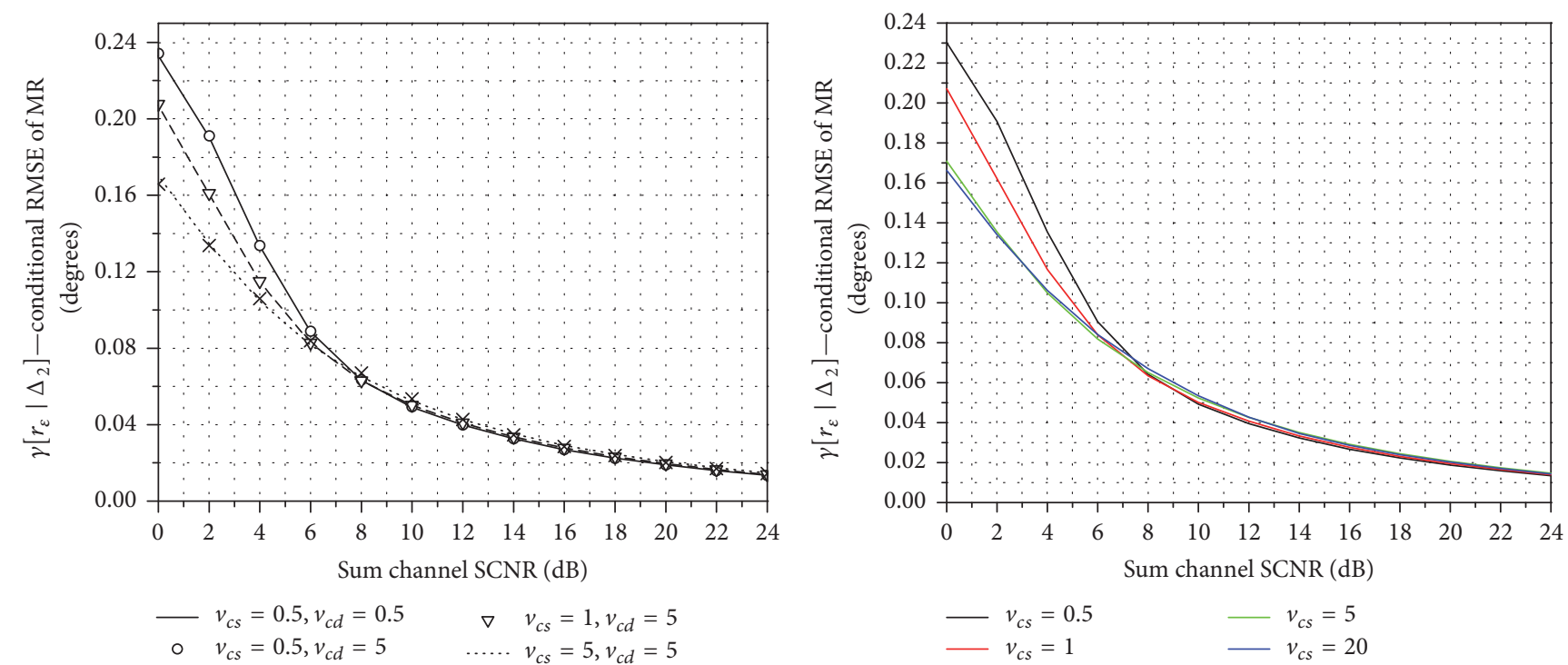

$$
\begin{aligned}
-v_{c s} & =0.5, v_{c d}=0.5 & \nabla & v_{c s}=1, v_{c d}=5 \\
\circ \quad v_{c s} & =0.5, v_{c d}=5 & \ldots \ldots & v_{c s}=5, v_{c d}=5 \\
---v_{c s} & =1, v_{c d}=1 & \times & v_{c s}=20, v_{c d}=20
\end{aligned}
$$

(a)

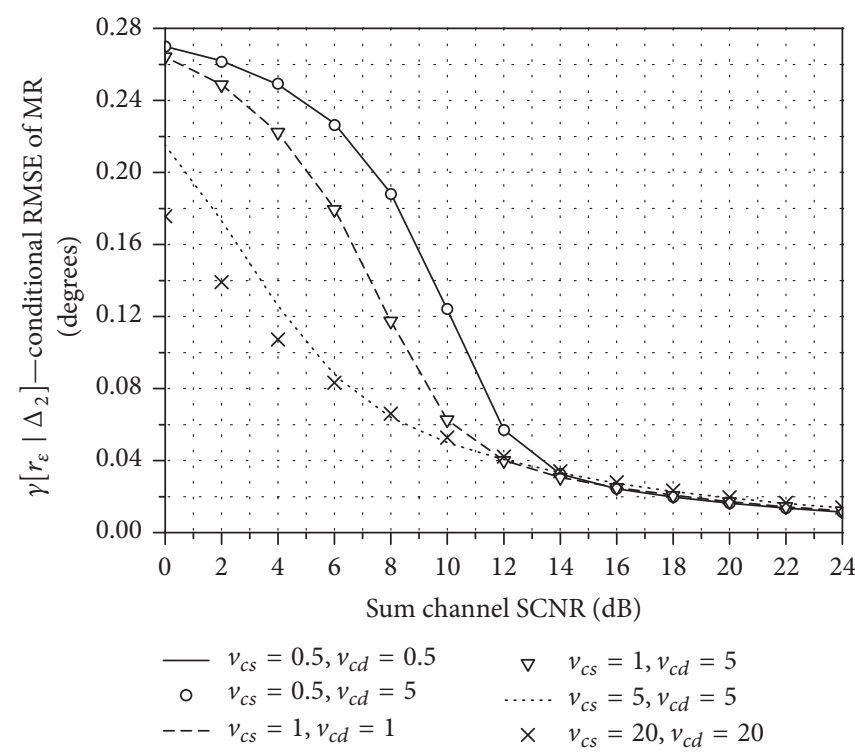

(c) $v_{c s}=1$

(b)

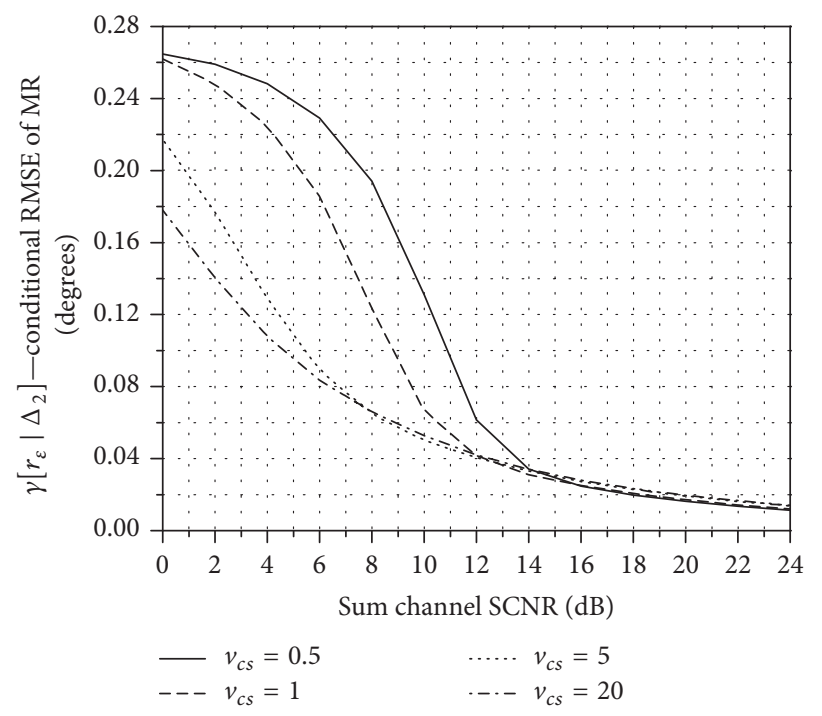

(d)

FIGURE 5: Angle measurement performance for different clutter shape parameters, for $\sigma_{n s}^{2}=1, P_{f}=10^{-4}, P_{\mathrm{opt}}=8$, and $\theta=0.3$; CNRs of sum and difference channels are $20 \mathrm{~dB}$ and $15 \mathrm{~dB}$, respectively. (a) Spatially independent texture case: $y_{s m}$ and $y_{d m}$ are independent. (b) Spatially independent texture case: $y_{s m}$ and $y_{d m}$ are completely correlated. (c) Spatially correlated texture case: $y_{s m}$ and $y_{d m}$ are independent. (d) Spatially correlated texture case: $y_{s m}$ and $y_{d m}$ are completely correlated.

shape parameter, the values of $\Upsilon\left[r_{\varepsilon} \mid \Delta_{2}\right]$ in the spatially correlated texture case are higher than those in the spatially independent texture case. Moreover, for low shape parameters, the decrease of $\Upsilon\left[r_{\varepsilon} \mid \Delta_{2}\right]$ with the increase of SCNR in the spatially correlated texture case is slow. Conversely, the comparisons of Figures 5(a) and 5(c) with Figures 5(b) and 5(d) indicate that even though the correlation properties of the texture components between the sum and difference channels are completely opposite, there are not the significant differences of $\Upsilon\left[r_{\varepsilon} \mid \Delta_{2}\right]$. This implies again that an increase in the spatial correlation of the texture components results in a degeneration of the angle measurement performance for the wideband monopulse radar, but the effects from the correlation of the texture components between the sum and difference channels are very feeble. If both four different correlation cases were considered simultaneously in the following simulations, the similar results should be observed. It is unnecessary that similar results are obtained repetitively. 


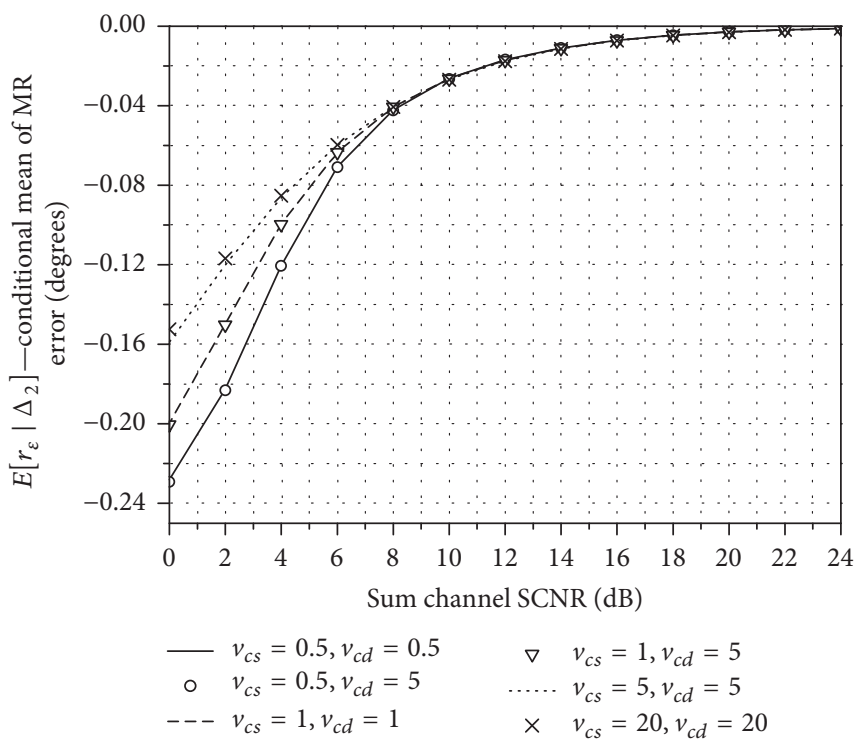

(a)

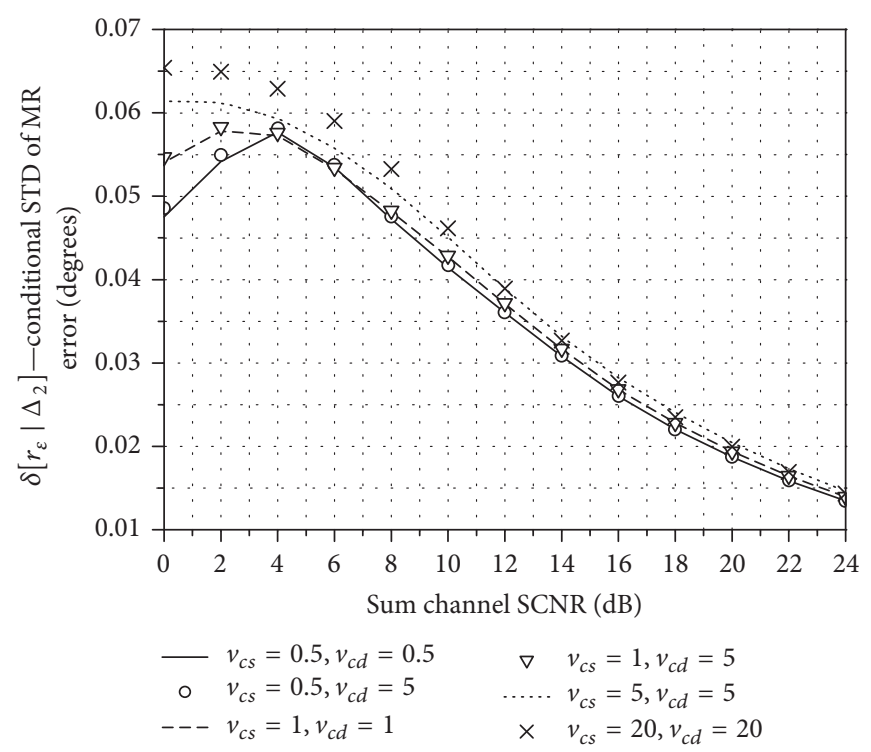

(b)

FIGURE 6: Conditional mean and conditional STD of MR error for different clutter shape parameters when the texture is independent in range and $y_{s m}$ and $y_{d m}$ are independent, where $\sigma_{n s}^{2}=1, P_{f}=10^{-4}, P_{\text {opt }}=8$, and $\theta=0.3$; CNRs of sum and difference channels are $20 \mathrm{~dB}$ and $15 \mathrm{~dB}$, respectively. (a) Conditional mean of MR error; (b) conditional STD of MR error.

Therefore, the following simulations are carried out only for the case where the texture components are spatially independent and $y_{s m}$ and $y_{d m}$ are independent.

4.2.3. Effect of the Correlation of the Speckle Components of Clutter. In high resolution radar system, for single range cell, the clutter of azimuth difference channel is uniformly distributed across all angle scope of the main lobes of difference pattern, but the off-boresight angle is bounded for the clutter of elevation difference channel due to the range sampling [1]. The clutter returns are modulated by the pattern gains of the difference antennas. Therefore, the characteristics of sea clutter are different between the elevation and the azimuth difference channels. Figure 7 shows the processed results of the measured clutter data, including the CNR and correlation curves. Three channels have the same noise power. In Figure 7(a), we can see that the shapes of CNR curves are similar between the sum and the azimuth difference channels, but the one of elevation difference channel is completely different. This agrees well with the theoretical analysis.

In addition, Figure 7 (c) shows that, in most range cells, the texture components between the sum and difference channels are almost completely correlated in elevation direction and are partially correlated in azimuth direction. However, there is a sharp drop in the correlation coefficients for both elevation and azimuth directions in middle range cells. In these range cells, the texture components are lowly correlated. Hence, it can be accepted in the preceding analysis that only two extremes cases (i.e., independent and completely correlated cases) are considered for the correlation between $y_{s m}$ and $y_{d m}$.
The results shown in Figure $7(\mathrm{~d})$ suggest that the speckle components of clutter between the sum and elevation difference channels are correlated, but those between the sum and azimuth difference channels are lowly correlated. It can be seen from (26), (27), and (31) that in the background of sea clutter plus noise the angle measurement performance is related to the correlation of the clutter speckle components between the sum and difference channels, as discussed in $[1,10-13]$ in the background of noise. Thus, Figure 8 is plotted to illustrate the effects of the correlation coefficients of the speckle components on the results of $\Upsilon\left[\begin{array}{lll}r_{\varepsilon} & \Delta_{2}\end{array}\right]$, $E\left[r_{\varepsilon} \mid \Delta_{2}\right]$, and $\sigma\left[r_{\varepsilon} \mid \Delta_{2}\right]$. According to (45) and (49) in the literature [10], the absolute value of $E\left[\begin{array}{l|l}r_{\varepsilon} & \Delta_{2}\end{array}\right]$ and $\sigma\left[r_{\varepsilon} \mid \Delta_{2}\right]$ decreases as correlation coefficient increases in the Gaussian noise environment. Similar results are also found for the $K$-distributed sea clutter plus noise environment, as shown in Figure 8. When SCNR is low, the performance improvement is significant by increasing the correlation coefficient. But this improvement can be ignored when SCNR is high. Furthermore, it should be noted that only the real part of the correlation of the speckle components can significantly affect the angle measurement performance, but the effects of the imaginary part are insignificant.

4.2.4. Effect of the Deviation Angle. When multiple targets occur in the measurement scene simultaneously, only one target among of them can be set at the radar beam boresight, and the others will deviate an angle from the boresight. Thereby, it is necessary to study the effects of deviation angle on angle measurement performance. The average powers of the target and of the clutter are modulated by the monopulse radar antenna gain patterns. For convenience, we assume 


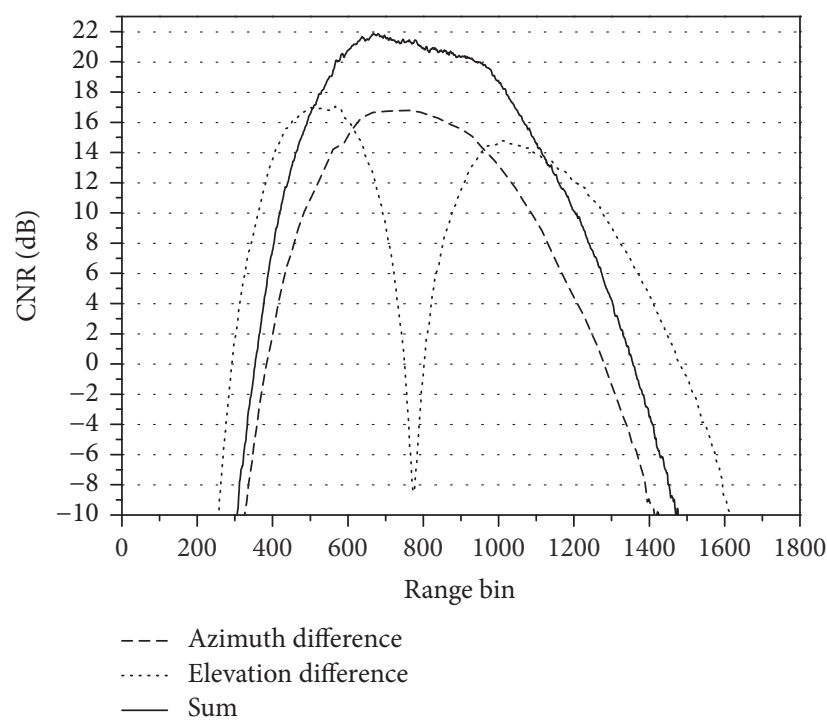

(a)

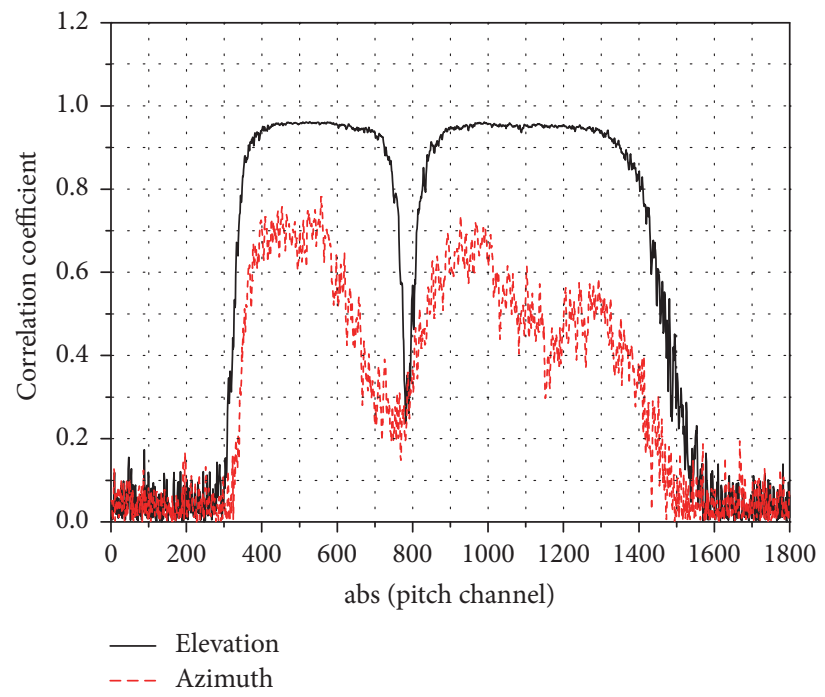

(c)

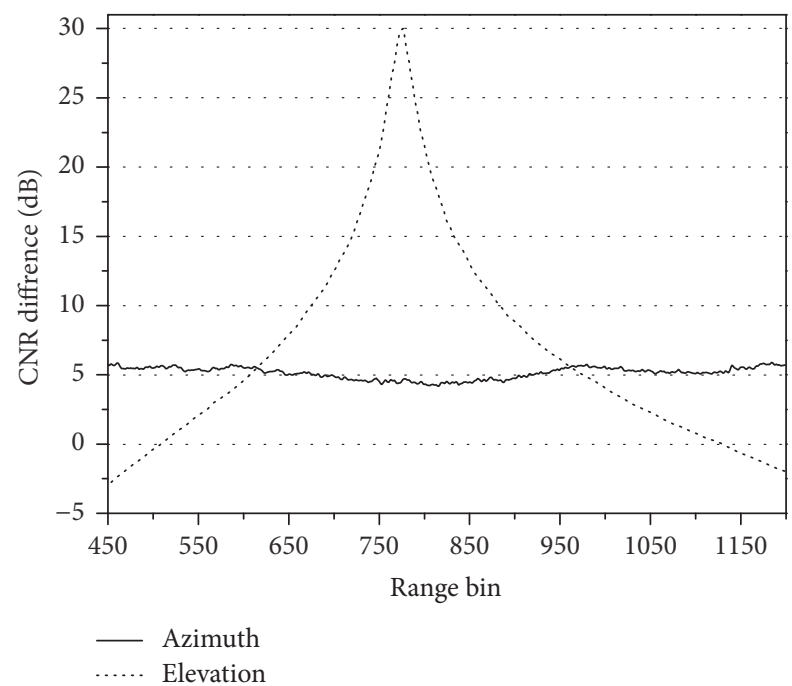

(b)

The correlation coefficient of speckle components between the elevation difference and sum channels

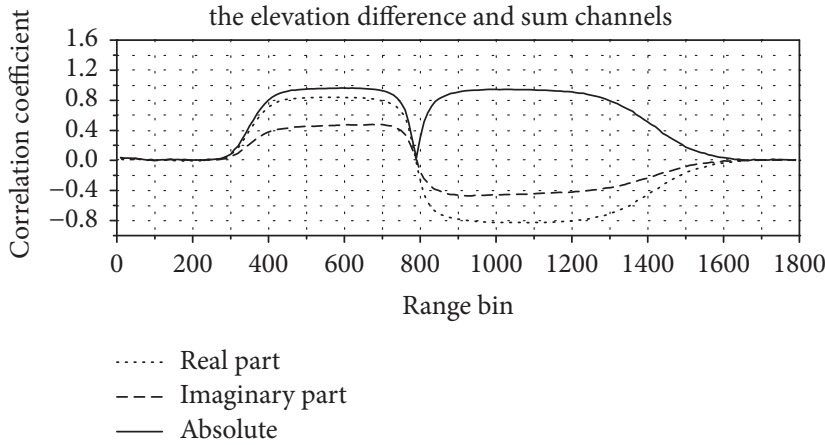

The correlation coefficient of speckle components between the azimuth difference and sum channels

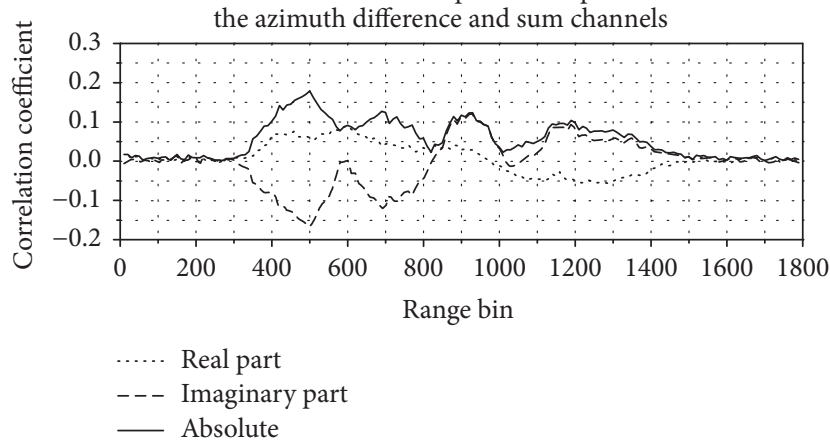

(d)

Figure 7: Measured clutter data processing results. (a) CNR of three channels; (b) CNR difference between the sum and difference channel; (c) the correlation coefficient of the texture components between the sum and difference channels; (d) the correlation coefficient of the speckle components between the sum and difference channels.

that while the elevation deviation angle varies, the azimuth deviation angle remains unchanged, and vice versa. $\bar{A}^{2}$ and $\sigma_{c s}^{2}$ denote the average powers of target and of clutter in the sum channel at the boresight, respectively. At the offboresight angle $\theta$, the average power of target in the sum channel becomes $\left[\left|g_{\Sigma}(\theta)\right|^{2} /\left|g_{\Sigma}(0)\right|^{2}\right] \bar{A}^{2}$ for both the elevation and azimuth directions. However, the changes of clutter power with the variation of the off-boresight angle in the elevation and azimuth directions are different. If in the elevation direction, the average power of sea clutter within the same range cell of target becomes $\left(\left|g_{\Sigma}(\theta)\right|^{2} /\left|g_{\Sigma}(0)\right|^{2}\right) \sigma_{c s}^{2}$ for the sum channel and $\left(\left|g_{\Delta}(\theta)\right|^{2} /\left|g_{\Delta}\left(\theta_{d}\right)\right|^{2}\right) \sigma_{c d}^{2}$ for the difference channel, respectively, where $\sigma_{c d}^{2}$ is the max clutter power in the elevation difference channel and is obtained 


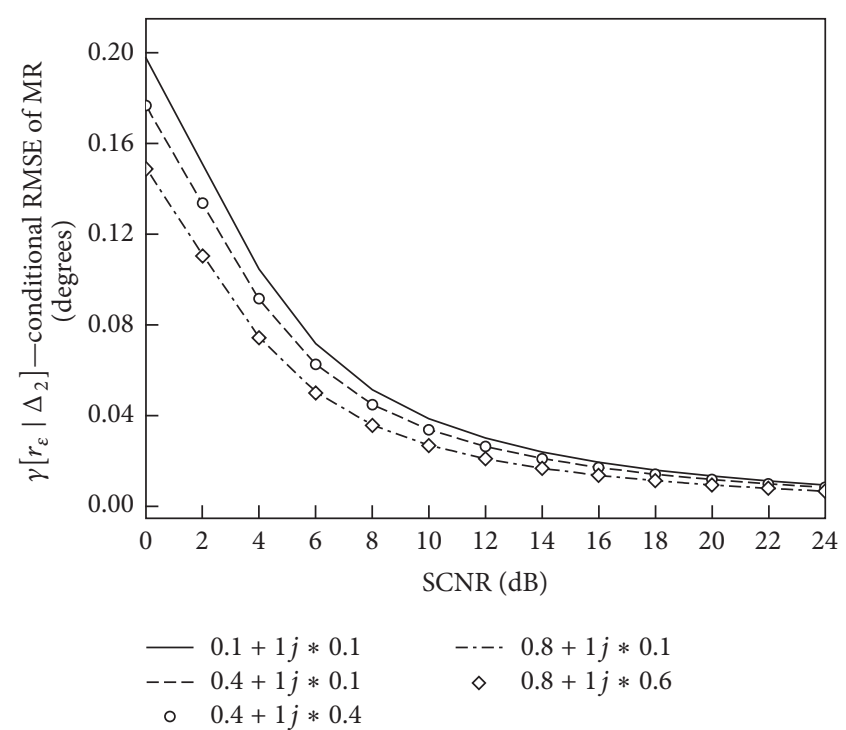

(a)

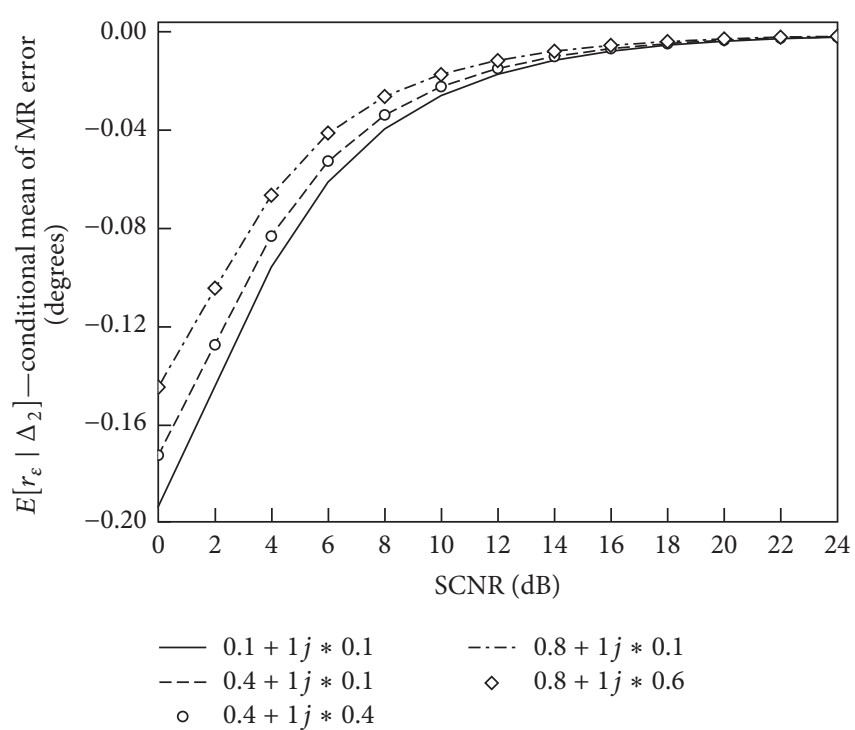

(b)

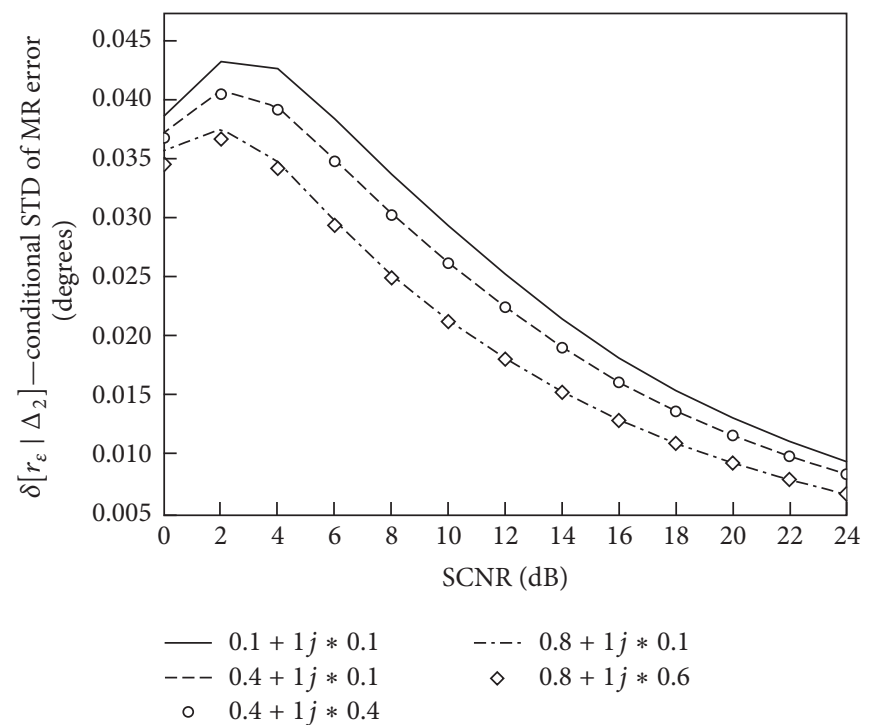

(c)

Figure 8: Performances of angle measurement in different correlation coefficients, for $v=1, \sigma_{n s}^{2}=1, P_{f}=10^{-4}, P_{\text {opt }}=8$, and $\theta=0.3$; CNRs of sum and difference channels are $20 \mathrm{~dB}$ and $15 \mathrm{~dB}$, respectively. (a) Conditional RMSE of MR; (b) conditional mean of MR error; (c) conditional standard deviation of MR error.

when the elevation deviation angle is $\theta_{d}$. But if in the azimuth direction, the average powers of sea clutter in the sum and difference channels always remain the initial power.

In Figure 9, $\Upsilon\left[r_{\varepsilon} \mid \Delta_{2}\right]$ is plotted against the deviation angle for different CNRs. In those simulations, the SCNR and CNR belong to the sum channel at the boresight. The CNR of azimuth difference channel is set to be the CNR of sum channel minus $5 \mathrm{~dB}$. The maximum $\mathrm{CNR}$ of elevation difference channel is also set to be the CNR of sum channel minus $5 \mathrm{~dB}$ when $\theta_{d}=0.55^{\circ}$.

From Figure 9, we can see that, in both the azimuth and elevation directions, $Y\left[\begin{array}{lll}r_{\varepsilon} & \mid & \Delta_{2}\end{array}\right]$ is monotonously increasing function of deviation angle. The target average power decreases as the deviation angle increases, resulting in the decrease of SCNR. Hence, the performance of angle measurement worsens with the increase of the deviation angle. As mentioned earlier, when the off-boresight angle occurs in the azimuth direction, the difference and sum channels CNRs do not vary. When the off-boresight angle occurs in the elevation direction, the difference and sum channel CNRs vary with variation of the deviation angle. Therefore, in Figure 9, the decreasing degree and speed of azimuth angle measurement performance are greater than those of elevation angle measurement performance. On the other hand, in Figure 9(a), the decreasing degrees of $\Upsilon\left[r_{\varepsilon} \mid \Delta_{2}\right]$ with the increase of the elevation deviation angle 


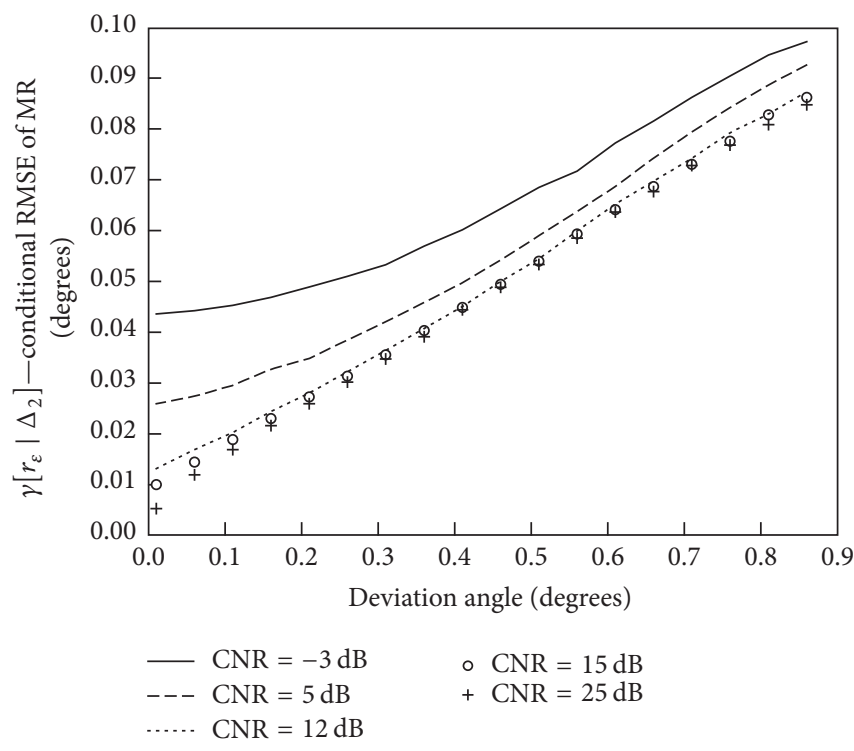

(a)

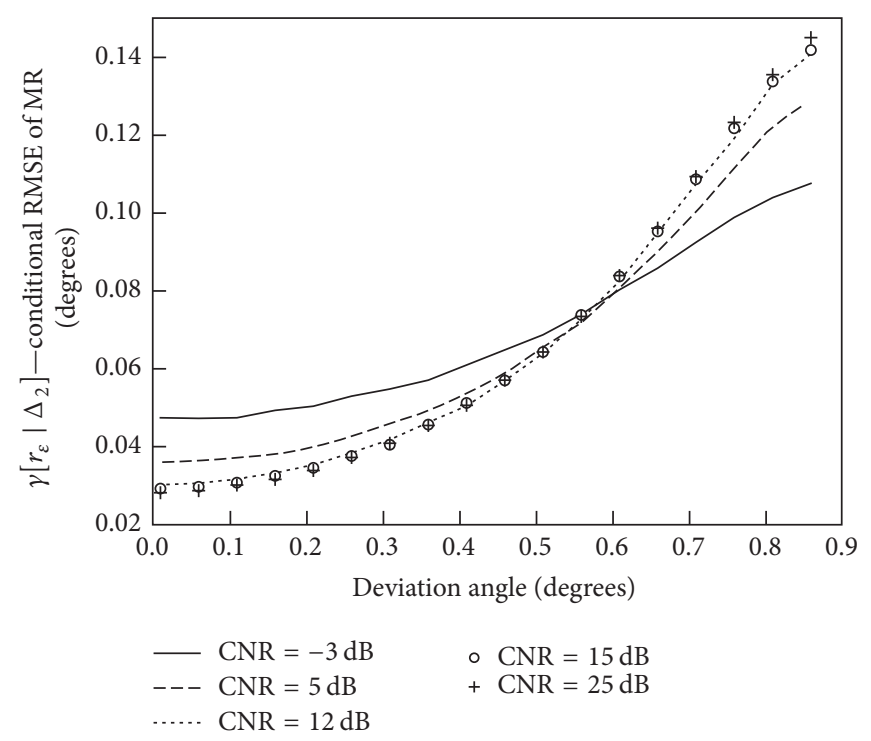

(b)

FIGURE 9: $\Upsilon\left[r_{\varepsilon} \mid \Delta_{2}\right]$ against the deviation angle in different CNRs, for SCNR $=12.6 \mathrm{~dB}, v=1, \sigma_{n s}^{2}=1, P_{f}=10^{-4}$, and $P_{\text {opt }}=8$. (a) Elevation direction; (b) azimuth direction.

are approximately equivalent for different CNRs. However, in Figure 9(b), the higher the CNRs are, the greater the decreasing degrees of $\Upsilon\left[r_{\varepsilon} \mid \Delta_{2}\right]$ with the increase of the azimuth deviation angle are.

\subsubsection{The Difference of the Effects between Sea Clutter and} Noise. The effects of sea clutter on monopulse angle measurement and those of noise are different. In the background of clutter plus noise, the change of CNR will cause the change of the angle measurement performance. To illustrate this, the angle measurement performances in different CNR cases are analyzed. In Figure 7(b), the CNR difference between the sum and difference channels in azimuth barely varies and is approximated to $5 \mathrm{~dB}$, whereas the one in elevation increases with the decrease of deviation angle and reaches the maximum on the boresight. When the elevation deviation angle is small, the CNR of sum channel is always bigger than those of both azimuth and elevation difference channels, and the CNR of elevation difference channel is much smaller than that of azimuth difference channel. Herein, the values of the CNR of sum channel minus $5 \mathrm{~dB}$ and $20 \mathrm{~dB}$ are set as the CNRs of azimuth and elevation difference channels, respectively. To avoid the effects of target deviation angle, it is assumed that the deviation angles of target in elevation and azimuth directions are the same. The results of $\Upsilon\left[r_{\varepsilon} \mid \Delta_{2}\right]$, $E\left[r_{\varepsilon} \mid \Delta_{2}\right]$, and $\sigma\left[r_{\varepsilon} \mid \Delta_{2}\right]$ are shown in Figure 10, where the values of CNR are sum channel clutters and the totals of clutter and noise powers are equal for all cases.

It can be clearly seen from Figures 10 (b) and 10(c) that, as the CNR increases, the absolute value of $E\left[r_{\varepsilon} \mid \Delta_{2}\right]$ increases and $\sigma\left[r_{\varepsilon} \mid \Delta_{2}\right]$ decreases. Moreover, when the target has low SCNR, the absolute values of $E\left[r_{\varepsilon} \mid \Delta_{2}\right]$ in high CNR cases are far larger than those in low CNR cases, but the values of $\sigma\left[\begin{array}{lll}r_{\varepsilon} & \Delta_{2}\end{array}\right]$ in high CNR cases are much smaller than those in low CNR cases. This confirms that the effects of sea clutter on monopulse angle measurement and those of noise are different. The noise mainly affects the standard divination of angle measurement error. The clutter has a "pulling" effect similar to that of an unresolved second target. It mainly affects the mean of angle measurement error. In particular, the clutter contributes an error mean that can be significant at low SCNR.

In addition, as the target SCNR increases, the decrease of $E\left[r_{\varepsilon} \mid \Delta_{2}\right]$ is very evident for the high CNR cases, whereas the decrease of $\sigma\left[r_{\varepsilon} \mid \Delta_{2}\right]$ is evident for the low CNR cases. When the target has high enough SCNR, there are close values of $E\left[\begin{array}{lll}r_{\varepsilon} & \Delta_{2}\end{array}\right]$ for all cases and these values have a tendency of approaching zero. However, under the same SCNR condition, $\sigma\left[r_{\varepsilon} \mid \Delta_{2}\right]$ still decreases as the CNR increases. Consequently, it is clearly shown in Figure 10(a) that $\Upsilon\left[r_{\varepsilon} \mid \Delta_{2}\right]$ increases with increasing CNR when the target has low SCNR, but it decreases when the target has high SCNR. In addition, Figure 10(a) suggests that there is a more serious angle estimation error for the high CNR cases when the target has low SCNR. As mentioned above, the maximum possible value of RMSE is of the order of the beamwidth. When the monopulse radar has a much wider beamwidth, the distance error due to the angle measurement error may easily reach the several lengths of the extended target in the high CNR case so that the measurement will be used to form a new track and steal the measurement of the existing track.

On the other hand, it can also be seen that the absolute values of $\Upsilon\left[r_{\varepsilon} \mid \Delta_{2}\right], E\left[\begin{array}{l|l}r_{\varepsilon} & \Delta_{2}\end{array}\right]$, and $\sigma\left[r_{\varepsilon} \mid \Delta_{2}\right]$ in elevation are smaller than those in azimuth. This is not surprising, since the sum channel returns are used together for the angle measurements in elevation and azimuth, and the CNR of elevation difference channel is lower than the one of azimuth difference channel around the boresight. 


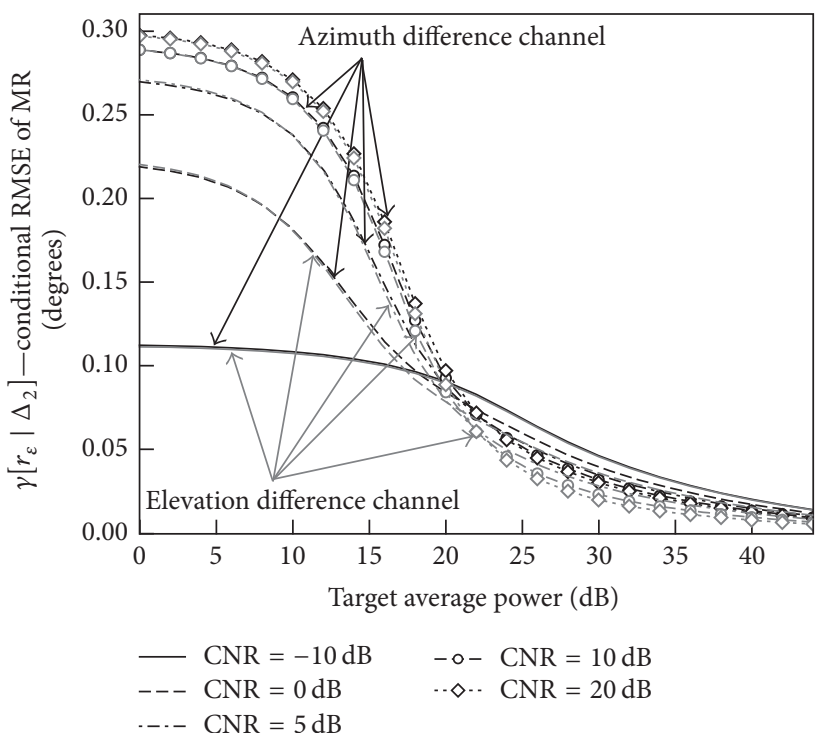

(a)

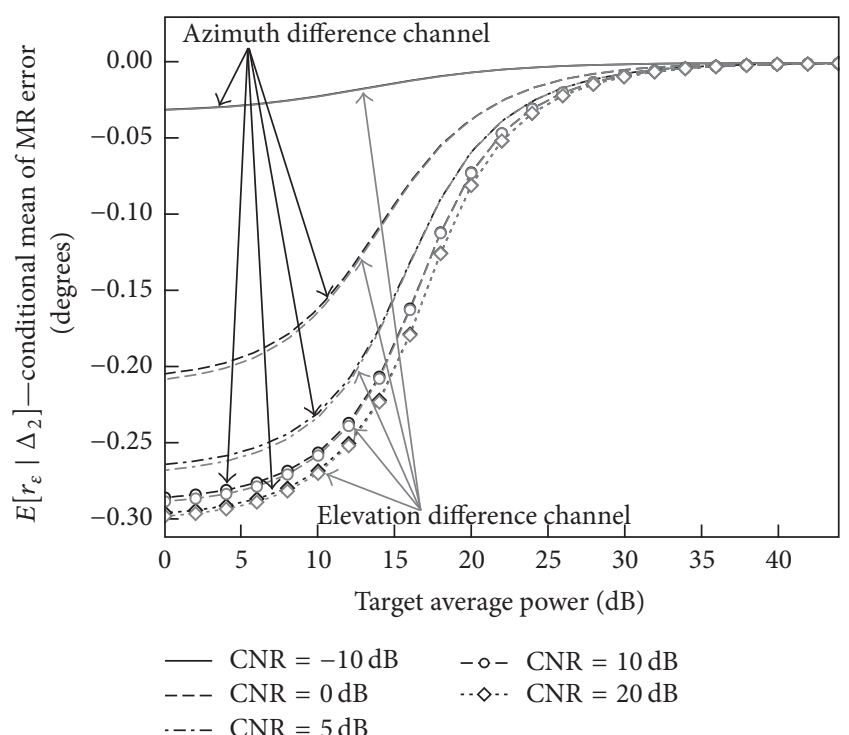

(b)

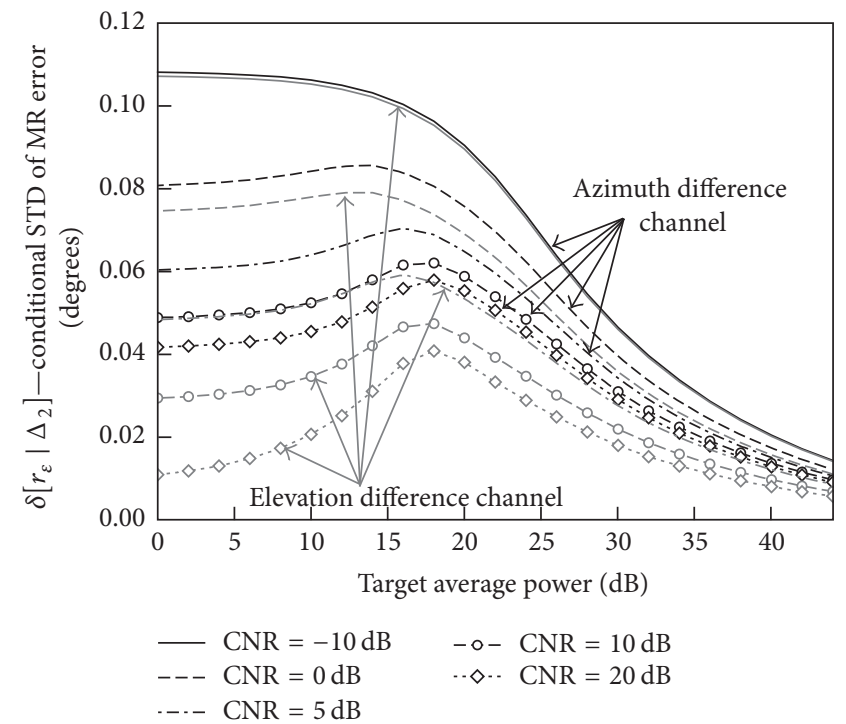

(c)

FIGURE 10: Performances of angle measurement for different clutter CNRs, for $v=1, P_{f}=10^{-4}, P_{\text {opt }}=8, \sigma_{c s}^{2}+\sigma_{n s}^{2}=15 \mathrm{~dB}$, and $\theta=0.3$. (a) Conditional RMSE of MR; (b) conditional mean of MR error; (c) conditional standard deviation of MR error.

Meanwhile, the deviation angles of target in elevation and azimuth are assumed to be the same. This indicates that the angle measurement in elevation is better than the one in azimuth when the elevation and azimuth deviation angles of target are the same and are small. However, although the CNR of azimuth difference channel is far higher than the one of the elevation channel, the differences of the performance between the elevation and azimuth angle measurements are not obvious.

\section{Conclusion}

In this paper, according to the angle measurement method with wideband monopulse radar and the binary detector, we deduce the conditional pdf of MR error for the targets on the sea. We consider both the spatial correlation of the texture components for each channel clutter and the correlation of the texture components between the sum and the difference channel clutter. For each correlation of the texture components, two bounding cases, namely, the independent and the completely correlated cases, are discussed. Furthermore, the modified experiential formula of the optimal parameter setting for binary detector is developed to apply in the background of $K$-distributed sea clutter plus noise. Then the effects of relevant parameters of clutter and detector on angle measurement performance are analyzed. Simulation results show the following.

(1) The effect of sea clutter on monopulse angle measurement performance is mainly reflected in affecting the angle-measuring bias and the effect of Gaussian noise 
on monopulse angle measurement performance is mainly reflected in affecting the angle-measuring standard deviation. The clutter contributes a bias that can be significant at low SCNR.

(2) The effect of $K$-distributed sea clutter on monopulse angle measurement performance is related to its shape parameter. The angle measurement performance weakens as shape parameter decreases when the SCNR of sum channel is low.

(3) The performance of angle measurement is optimal at the boresight. It weakens as deviation angle decreases. The performance deterioration of detection and angle measurement are more serious in azimuth than elevation.

(4) The effects of the correlation between the sum and difference channel clutter on monopulse angle measurement performance include two aspects: the effects from the correlation of the texture components are very feeble, and an increase in the correlation of the speckle components will result in an improvement of the angle measurement performance.

(5) The increase in spatial correlation of the texture components will result in a degeneration of the angle measurement performance for the wideband monopulse radar.

For any radar designer, it is a great challenge to detect and track the sea surface target by using the wideband monopulse radar. Our work provides a comprehensive understanding of the effect of the design of wideband monopulse radar on the angle measurement performance in $K$-distributed sea clutter plus noise. In this paper, we do not consider the effects of multipath and flare angle of target. We plan to study these issues as a future work.

\section{Conflicts of Interest}

The authors declare that there are no conflicts of interest regarding the publication of this paper.

\section{References}

[1] S. M. Sherman, Monopulse Principles and Techniques, Artech House, Inc, 2nd edition, 2011.

[2] E. Daeipour, W. D. Blair, and Y. Bar-Shalom, "Bias compensation and tracking with monopulse radars in the presence of multipath," IEEE Transactions on Aerospace and Electronic Systems, vol. 33, no. 3, pp. 863-882, 1997.

[3] H. Z. Zhao, "High resolution profile-based angular tracking technology for monopulse radar," Acta Electronica Sinica, vol. 28, no. 4, pp. 1-4, 2000.

[4] T. Zhang, Q. Zhang, C. Z. Ma, and S. H. Zhang, "A study of the suppression of the angle glint based on high resolution profile," Journal of Xidian University, vol. 28, no. 3, pp. 296-300, 2001.

[5] Q. Li, S. H. Zhang, H. Y. Zhang, and Z. Liu, "Maximum entropy method for angle estimation in wideband monopulse tracking radar," Acta Electronica Sinica, vol. 34, no. 12, pp. 2180-2184, 2006.

[6] J. H. Liu, X. H. Zheng, and L. Sun, "Analysis of simulation on the active seeker low altitude multipath problem," Modern Defence Technology, vol. 35, no. 3, pp. 82-85, 2007.
[7] K. Haspert and M. Tuley, "Comparison of predicted and measured multipath impulse responses," IEEE Transactions on Aerospace and Electronic Systems, vol. 47, no. 3, pp. 1696-1709, 2011.

[8] A. Sinha, Y. Bar-Shalom, W. D. Blair, and T. Kirubarajan, "Radar measurement extraction in the presence of sea-surface multipath," IEEE Transactions on Aerospace and Electronic Systems, vol. 39, no. 2, pp. 550-567, 2003.

[9] Y. Yang, D.-J. Feng, X.-S. Wang, and S.-P. Xiao, "Effects of k distributed sea clutter and multipath on radar detection of low altitude sea surface targets," IET Radar, Sonar and Navigation, vol. 8, no. 7, pp. 757-766, 2014.

[10] A. D. Seifer, "Monopulse-radar angle tracking in noise or noise jamming," IEEE Transactions on Aerospace and Electronic Systems, vol. 28, no. 3, pp. 622-638, 1992.

[11] A. D. Seifer, "Monopulse-radar angle measurement in noise," IEEE Transactions on Aerospace and Electronic Systems, vol. 30, no. 3, pp. 950-957, 1994.

[12] G. W. Groves, W. Dale Blair, and W. C. Chow, "Probability distribution of complex monopulse ratio with arbitrary correlation between channels," IEEE Transactions on Aerospace and Electronic Systems, vol. 33, no. 4, pp. 1345-1350, 1997.

[13] E. Chaumette and P. Larzabal, "Statistical prediction of monopulse angle measurement," IET Radar, Sonar and Navigation, vol. 1, no. 5, pp. 377-387, 2007.

[14] K. D. Ward, R. J. A. Tough, and S. Watts, Sea Clutter: Scattering, The K Distribution and Radar Performance, IET, London, UK, 2nd edition, 2013.

[15] E. Conte, A. De Maio, and C. Galdi, "Statistical analysis of real clutter at different range resolutions," IEEE Transactions on Aerospace and Electronic Systems, vol. 40, no. 3, pp. 903-918, 2004.

[16] M. Greco, F. Gini, and M. Rangaswamy, "Statistical analysis of measured polarimetric clutter data at different range resolutions," IEE Proceedings: Radar, Sonar and Navigation, vol. 153, no. 6, pp. 473-481, 2006.

[17] Y. Yang, S.-P. Xiao, D.-J. Feng, and W.-M. Zhang, "Modelling and simulation of spatial-temporal correlated $\mathrm{K}$ distributed clutter for coherent radar seeker," IET Radar, Sonar and Navigation, vol. 8, no. 1, pp. 1-8, 2014.

[18] F. AluffiPentini, A. Farina, and F. Zirilli, "Radar detection of targets located in a coherent K distributed clutter background," IEE Proceedings F (Radar and Signal Processing), vol. 139, no. 3, pp. 239-244, 1992.

[19] E. Conte and G. Ricci, "Performance prediction in compoundgaussian clutter," IEEE Transactions on Aerospace and Electronic Systems, vol. 30, no. 2, pp. 611-616, 1994.

[20] B. C. Armstrong and H. D. Griffiths, "CFAR detection of fluctuating targets in spatially correlated K-distributed clutter," IEE Proceedings F (Radar and Signal Processing), vol. 138, pp. 139-151, 1991.

[21] S. D. Blunt, K. Gerlach, and J. Heyer, "HRR detector for slowmoving targets in sea clutter," IEEE Transactions on Aerospace and Electronic Systems, vol. 43, no. 3, pp. 965-974, 2007.

[22] H. Z. Zhao, "Optimal binary detection strategy for rangeextended targets in sea clutter," Systems Engineering and Electronics, vol. 33, no. 5, pp. 982-986, 2011.

[23] Y. Z. Chen, "Optimal binary detection for distributed targets," Systems Engineering and Electronics, vol. 33, no. 1, pp. 26-29, 2011. 
[24] D. Wu, D. Zhu, M. Shen, and Y. Li, "Statistical analysis of monopulse-synthetic aperture radar for constant false-alarm rate detection of ground moving targets," IET Radar, Sonar and Navigation, vol. 9, no. 6, pp. 641-652, 2015.

[25] S. Watts, "Cell-averaging CFAR gain in spatially correlated K-distributed clutter," IEE Proceedings - Radar, Sonar and Navigation, vol. 143, no. 5, pp. 321-327, 1996.

[26] A. Papoulis and S. U. Pillai, Probability, Random Variables and Stochastic Processes, McGraww-Hill, New York, NY, USA, 4th edition, 2002.

[27] S. Watts, "Radar detection prediction in sea clutter using the compound K -distribution model," IEE Proceedings, vol. 132, no. 7, pp. 613-620, 1985.

[28] M. A. Richards, J. A. Scheer, and W. A. Holm, Principles of Modern Radar, Volume 1, Basic Principles, SciTech Publishing Company, 2010. 


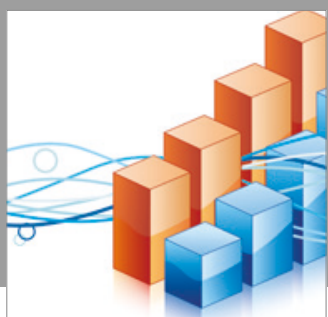

Advances in

Operations Research

vatersals

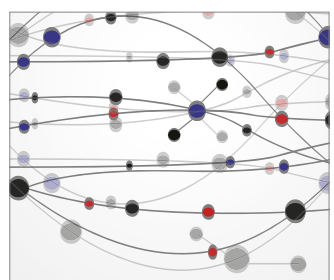

\section{The Scientific} World Journal
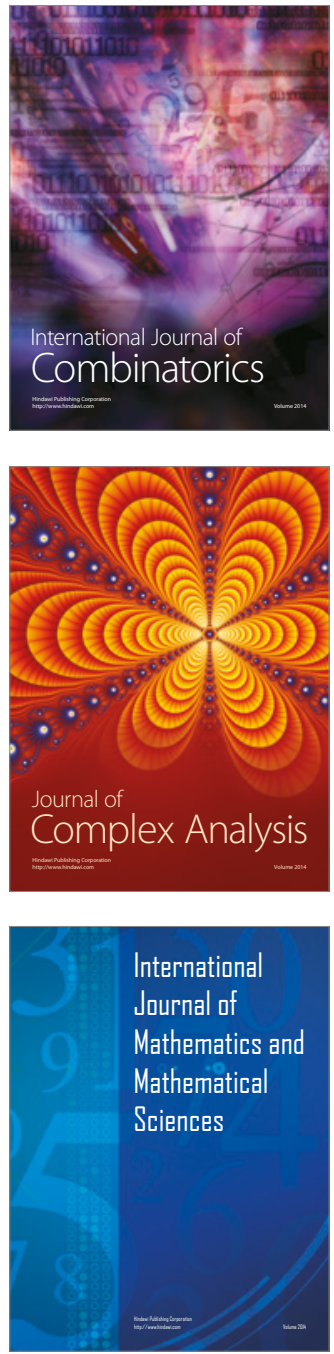
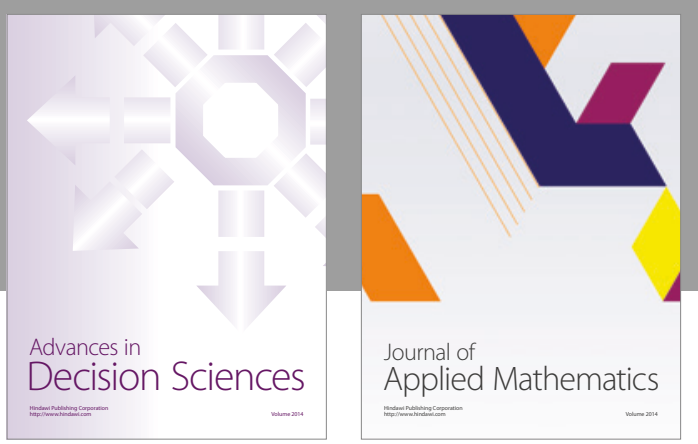

Algebra

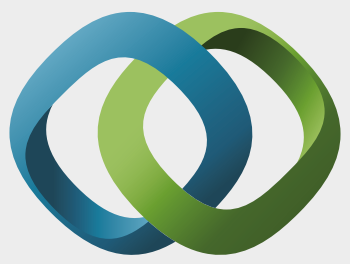

\section{Hindawi}

Submit your manuscripts at

https://www.hindawi.com
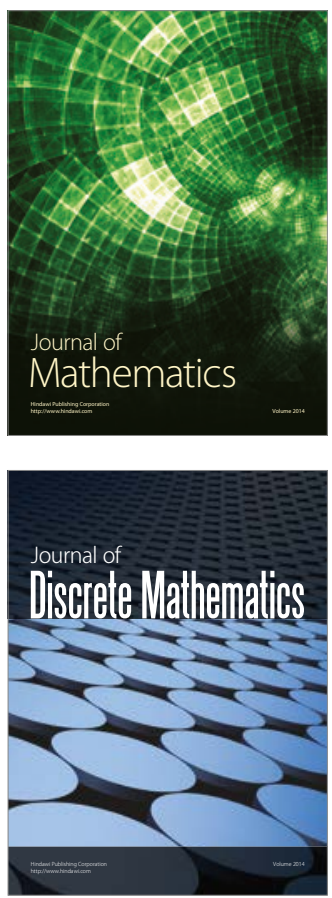

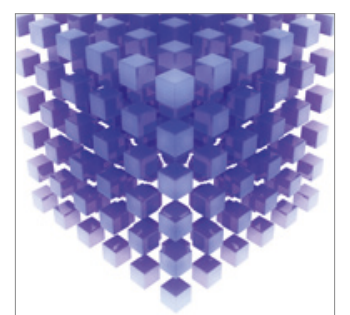

Mathematical Problems in Engineering
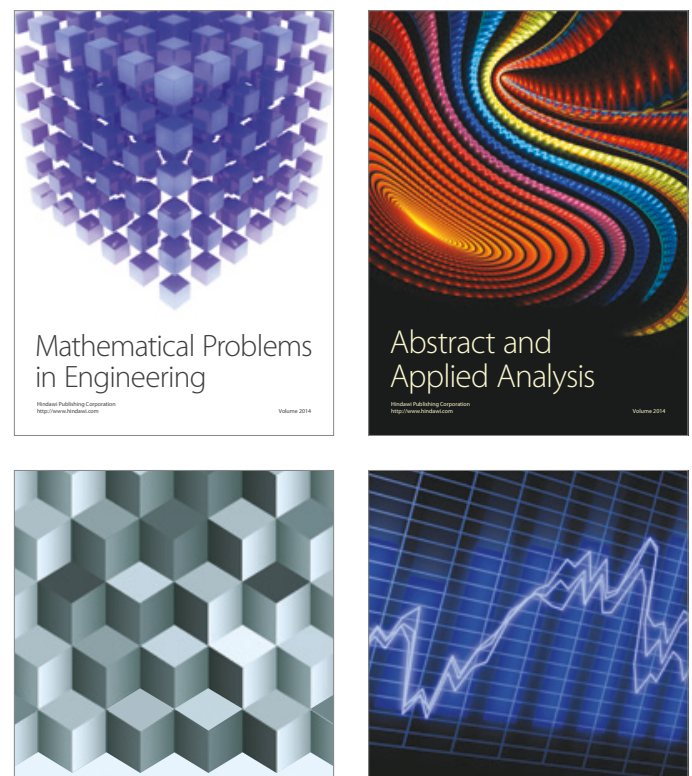

Journal of

Function Spaces

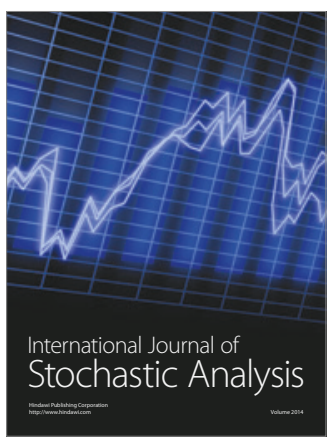

Probability and Statistics
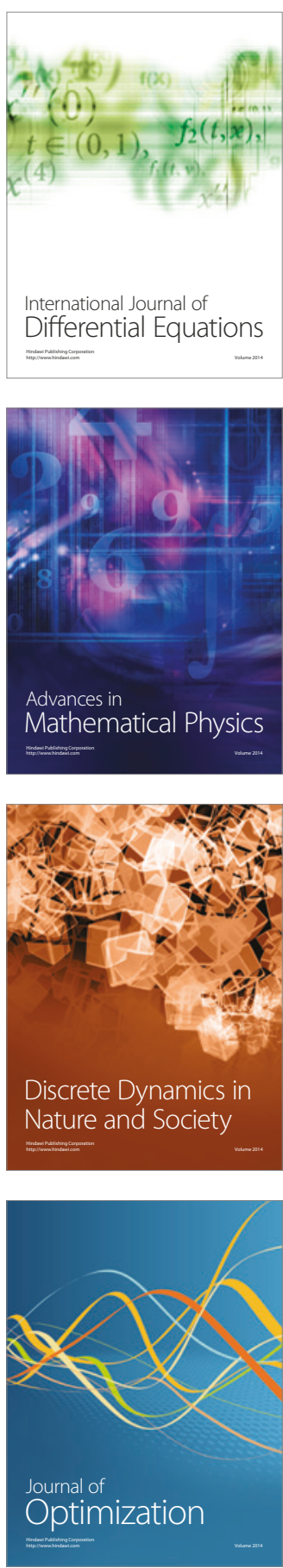